Related content

\title{
Characterization of the structure and eigenvalue spectra of the compound states of Sm IX
}

To cite this article: D Kilbane et al 2006 Phys. Scr. 73198

- Signatures of quantum chaosin rare earth
elements: I
A Cummings, G O'Sullivan and D M
Heffernan
- Signatures of quantum chaos in rare earth
$\frac{\text { elements:II }}{\text { A Cummings, G O'Sullivan and D M }}$
Heffernan
- Mixing of dielectronic and multiply excited
$\frac{\text { States }}{\text { G F Gribakin and S Sahoo }}$

View the article online for updates and enhancements.

\section{Recent citations}

$$
\begin{aligned}
& \text { Ground-state configurations and } \\
& \frac{\text { theoretical soft-x-ray emission of highly }}{\text { charged actinide ions }} \\
& \text { J. Sheil et al }
\end{aligned}
$$




\title{
Characterization of the structure and eigenvalue spectra of the compound states of Sm IX
}

\author{
D Kilbane ${ }^{1,2}$, A Cummings ${ }^{1}$, D M Heffernan ${ }^{2}$ and G O'Sullivan $^{1}$ \\ ${ }^{1}$ Department of Mathematical Physics, National University of Ireland, Maynooth, Ireland \\ ${ }^{2}$ UCD School of Physics, University College Dublin, Belfield, Dublin 4, Ireland \\ E-mail: deirdre.kilbane@dcu.ie
}

Received 25 August 2005

Accepted for publication 20 September 2005

Published 17 January 2005

Online at stacks.iop.org/PhysScr/73/198

\begin{abstract}
Using the configuration interaction Hartree-Fock method, the even and odd Hamiltonian matrices of Sm IX are studied in the $l s$ and $j j$ coupling schemes. Sm IX is isoelectronic with Xe but because of $4 \mathrm{f}$ orbital contraction, and the near degeneracy of $4 \mathrm{f}$ and $5 \mathrm{p}$ binding energies, the lowest configuration is a mixture of states with differing numbers of $4 \mathrm{f}$ and $5 \mathrm{p}$ electrons. Diagonalization of the Hamiltonian results in a set of eigenvalues and eignvectors which are characterized using various statistical tests of random matrix theory.

PACS numbers: 05.45.Mt, 31.10.+z, 31.15.-p
\end{abstract}

(Some figures in this article are in colour only in the electronic version.)

\section{Introduction}

Random matrix theory (RMT) was developed by Wigner $[1,2]$ to model the statistical distributions of eigenvalues and eigenfunctions (EFs) of complex many-body quantum systems. Initially, applications of RMT were confined to nuclear physics where the theory was successful in describing the spectral fluctuation properties of atomic nuclei. Rosenzweig and Porter [3] analysed spectra of neutral atoms which constituted the first application of RMT outside the field of nuclear physics and provided the first strong evidence for the universal applicability of RMT.

However, RMT was found to be quite limited as it can only give a proper description of a system locally in a restricted region of the energy spectra. New approaches in RMT have been developed by imposing internal structure on random matrices. One such approach is band random matrices (BRMs) [4-8], where the basis states are ordered in such a way that a Hamiltonian matrix has a band of nonzero matrix elements $H_{i j}$ interconnecting the states within the band $|i-j| \leqslant b$ around a main diagonal. Inside the band, the matrix elements are assumed to be random and independent, and outside the band, matrix elements are set to zero.

In an atomic system, the Hamiltonian matrix can be viewed as $H=H_{0}+V$, where $H$ can be described as a banded matrix with a leading diagonal with monotonically increasing values, plus random and independent off-diagonal elements inside a band of size $b-\mathrm{a}$ Wigner BRM (WBRM) [1, 2]. The basis states of $H$ are many-particle eigenstates of the mean field $H_{0}$, and are composed of non-interacting single-particle states (eigenstates of the same $J^{\pi}$, angular momentum and parity only, constitute a given spectrum). When the perturbation (configuration interaction (CI)) is switched on, the unperturbed many-particle states are coupled together and form compound atomic states [9], with properties analogous to nuclear compound states, due to the large density of states and the small average level spacing $(D)$. This coupling therefore gives rise to correlations between the eigenvector components $C_{i j}$ of $H$, and to correlations between the energy eigenvalues of $H$, with the degree of correlation depending on the size of the band, $b$, of $V$ and on the size of the matrix elements of $V$. Thus the application of $V$ can give rise to different types of energy level statistics. The ratio of the typical off-diagonal matrix element to the average level spacing $V / D$ defines the perturbative $(V / D<1)$ or non-perturbative $(V / D \geqslant 1)$ and possibly chaotic character of the mixing states [14].

Flambaum et al [9-13], Gribakin et al [14, 15] and Zelevinsky et al [16] have done pioneering work in this area, producing a realistic model of a quantum chaotic system by characterizing the spectrum and eigenstates of the 
Structure and eigenvalue spectra of the compound states of Sm IX

lowest states with $J^{\pi}=4^{-}$of the rare-earth atom Ce. These studies were extended to include praseodymium $J^{\pi}=\frac{11}{2}^{ \pm}$by Cummings et al [17, 18], where the Hamiltonian and coupling matrices, energy eigenvalues and dipole moments of Pr I were also investigated, with possible multifractal behaviour found in several rare-earth elements including Ce [19].

Of particular interest however is the situation in more highly ionized, heavier rare-earth ions that contain $4 \mathrm{f}$ and $5 \mathrm{p}$ electrons in their ground configurations. In stages past six or seven times ionized there is a near degeneracy in the binding energies of $4 \mathrm{f}$ and $5 \mathrm{p}$ electrons resulting from the tendency of levels to regroup according to principal quantum numbers with increasing ionization. Initially, in the neutral lanthanides, $4 \mathrm{f}$ electrons are more weakly bound than either the $5 \mathrm{p}$ or $5 \mathrm{~s}$, but by the 15 th ion stage lie lower than both [20, 21]. Such behaviour gives rise to the so-called hyperalkali ions where $4 \mathrm{f}^{6} 5 \mathrm{p}^{6} 5 \mathrm{~s}^{2} 5 \mathrm{~d}$ becomes $4 \mathrm{f}^{14} 5 \mathrm{~s}$ along the $\mathrm{Pm}$ isoelectronic sequence [22-24]. At intermediate stages, where the 5p and $4 \mathrm{f}$ energies are very close, there is a vast increase in the number of available levels due to the appearance of a whole range of configurations based on variable $4 \mathrm{f} / 5 \mathrm{p} / 5 \mathrm{~s}$ occupancy lying well below the ionization limit. There is in this region, a complete breakdown of the single-particle approximation and these configurations are so highly mixed that single-electron labels are no longer appropriate. In fact, the configurations mix to form 'superconfigurations'.

In an earlier work on this problem, it was shown that along the Xe I sequence, the various $(4 \mathrm{f} 5 \mathrm{p})^{6}$ configurations are closest in Sm IX [25]. Experimentally, no lines are observed either in the spectrum of this series or adjacent ions, so that in fact the emission from a samarium plasma containing ion stages from VI to XIV consists of a pure continuum extending from 3 to $200 \mathrm{~nm}$ [26]. From a theoretical analysis of some of the level distributions in this and adjacent ions, it was found that the level spacings followed a Wigner distribution and possessed many of the attributes associated with the emergence of chaotic behaviour. In the present work, we repeat some of these tests for a more extensive basis set and perform a range of additional tests for the presence of chaos. Because of the enormous level densities encountered, the resulting number of transitions is so great that in many cases, the line spacing is less than the linewidth of individual lines so that the emission appears continuous on all length scales, unless one is dealing with an isolated ion or a small number of ions for a limited time.

In order to extend on our previous work [27], detailed calculations were performed with the Cowan suite of atomic codes [28], using the configuration interaction Hartree-Fock (HFCI) method. For the $J=0 \rightarrow J=1$ transitions, the configurations considered are given in table 1 . These were chosen as they lie closest in energy. From a single-configuration calculation, the lowest configuration is $5 s^{2} 5 p^{4} 4 f^{2}$. Excitation of $5 p-5 d$ or $5 s-5 p$ requires essentially the same energy. Because of memory constraints we omitted 'correlations' in the form of additional high-lying configurations, though to some extent such interactions are implicitly allowed for through the scaling of Slater-Condon parameters used in these calculations. From a comparison between our calculated values and experimentally observed energies of $4 \mathrm{f} \rightarrow 5 \mathrm{~d}$ transitions in Ag I-like Sm XVI, the accuracy of the present calculations is inferred to be better than $1 \%$ [29].
Table 1. The even and odd configurations of Sm IX.

\begin{tabular}{lll}
\hline & Even & Odd \\
\hline Configurations & $5 \mathrm{~s}^{2} 5 \mathrm{p}^{4} 4 \mathrm{f}^{2}$ & $5 \mathrm{~s}^{2} 5 \mathrm{p}^{3} 4 \mathrm{f}^{2} 5 \mathrm{~d}$ \\
& $5 \mathrm{~s}^{2} 5 \mathrm{p}^{3} 4 \mathrm{f}^{3}$ & $5 \mathrm{~s}^{2} 5 \mathrm{p}^{2} 4 \mathrm{f}^{3} 5 \mathrm{~d}$ \\
& $5 \mathrm{~s}^{2} 5 \mathrm{p}^{2} 4 \mathrm{f}^{4}$ & $5 \mathrm{~s}^{2} 5 \mathrm{p}^{1} 4 \mathrm{f}^{4} 5 \mathrm{~d}$ \\
& & $5 \mathrm{~s}^{1} 5 \mathrm{p}^{5} 4 \mathrm{f}^{2}$ \\
& & $5 \mathrm{~s}^{1} 5 \mathrm{p}^{4} 4 \mathrm{f}^{3}$ \\
& & $5 \mathrm{~s}^{1} 5 \mathrm{p}^{3} 4 \mathrm{f}^{4}$ \\
$E_{\text {ave }}$ range $(\mathrm{eV})$ & $0 \rightarrow 5$ & $30 \rightarrow 40$ \\
Possible $J$ values & $0 \rightarrow 12$ & $0 \rightarrow 14$ \\
\hline
\end{tabular}

In section 2, the Hamiltonian matrices are characterized. Section 3 details the structure of the eigenstates, which shows clearly the breakdown of the single-particle model, and finally section 4 analyses the energy level statistics. Thus, using the arguments of $[9,16,17]$ we extend the model for chaotic quantum systems further along the rare-earth sequence into a region of high complexity by analysing the eigenvalue spectra and eigenstates of Sm IX.

\section{Banded Hamiltonian matrices}

In this section, the banded structure of the Hamiltonian matrix is investigated following the systematic analysis of $[9,12,16$, $17,30]$.

\subsection{Hamiltonian matrix element distribution}

The window averaged $\left\langle H^{2}\right\rangle_{i j}$, shown in figure 1 , is used to show the bandedness of the Hamiltonian matrices [9, 12],

$$
\left\langle H^{2}\right\rangle_{i j}=\frac{1}{(2 W+1)^{2}} \sum_{\substack{\left|i^{\prime}-i\right| \leqslant W \\\left|j^{\prime}-j\right| \leqslant W}} H_{i^{\prime} j^{\prime}}^{2}
$$

where $W=10$ is the size of the averaging window chosen for Sm IX. The square of the Hamiltonian matrix elements was averaged in such a way that there is now a reduced matrix $N / 10 \times N / 10$. The basis states are arranged so that the diagonalized $H$ has its $H_{i j}$ matrix elements increasing monotonically with $i$.

The diagonal is more pronounced in both the $l s$ and $j j$ coupling schemes of even Sm IX than odd Sm IX. For even Sm IX, the amplitude of these effective matrix elements decreases when moving away from the diagonal. Therefore, the structure of the Hamiltonian can be treated as band-like, although it is clear that the amplitude of the averaged matrix elements decays quite slowly. The situation is similar for odd Sm IX although there are some large off-diagonal matrix elements present.

\subsection{Off-diagonal elements of the Hamiltonian matrices}

The dependence of the off-diagonal matrix elements $H_{i j},(i \neq$ $j$ ), on the level numbers $i, j$ is almost random due to the basis states $\psi_{i}$ being 'complex' linear combinations of the singledeterminant states. Statistics characterizing the off-diagonal elements of the Hamiltonian matrices for the even and odd levels of Sm IX are presented in tables 2 and $3 . W$ is the number of elements of the 'upper triangle' of the matrix. In this work, $\left|H_{i j}\right|$ was set to 0 if $\left|H_{i j}\right| \leqslant 10^{-3} \mathrm{eV}$. 

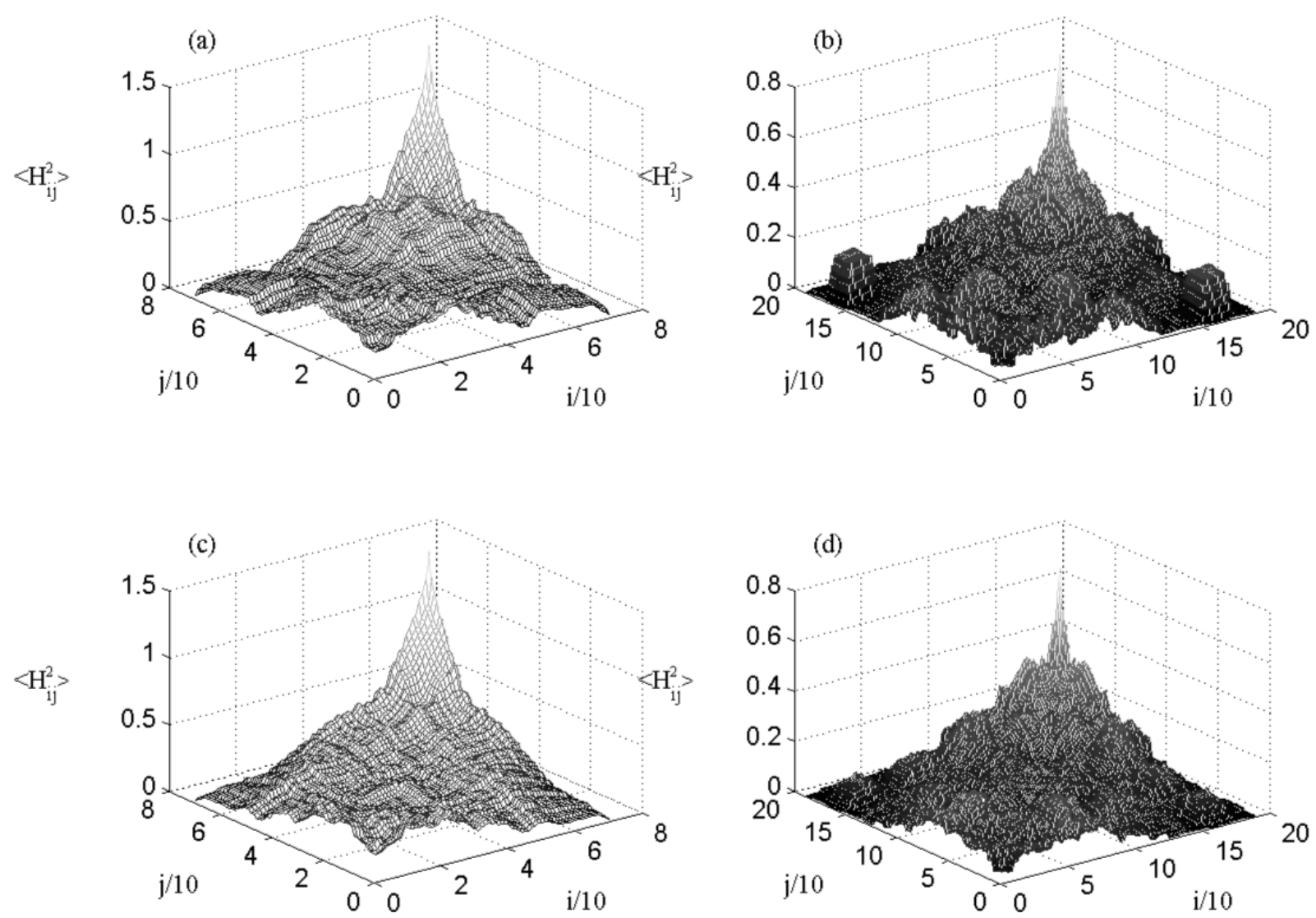

Figure 1. Window-averaged Hamiltonian matrix for (a) even Sm IX in $j j$ coupling, (b) odd Sm IX in $j j$ coupling, (c) even Sm IX in $l s$ coupling and (d) odd Sm IX in $l s$ coupling.

Table 2. Statistical characteristics of the off-diagonal Hamiltonian matrix elements of even Sm IX in $l s$ and $j j$ coupling.

\begin{tabular}{lll}
\hline & Whole matrix & $H_{i j} \neq 0$ only \\
\hline Sm IX even $l s$ & & \\
$\overline{H_{i j}^{2}}\left(\mathrm{eV}^{2}\right)$ & 0.284 & 0.869 \\
$\sqrt{\overline{H_{i j}^{2}}}(\mathrm{eV})$ & 0.533 & 0.932 \\
$H_{i j}(\mathrm{eV})$ & $-2.57 \times 10^{-2}$ & $-7.88 \times 10^{-2}$ \\
No of $H_{i j}(i<j)$ & 4095 & 1336 \\
Matrix dimension & $91 \times 91$ & \\
$D(\mathrm{eV})$ & & 0.31 \\
$\frac{V}{D}$ & & 3.009 \\
$\mathrm{Sm} \mathrm{IX} \mathrm{even} j j$ & & \\
$\overline{H_{i j}^{2}}(\mathrm{eV})$ & 0.282 & 0.533 \\
$\sqrt{\overline{H_{i j}^{2}}}(\mathrm{eV})$ & 0.531 & 0.730 \\
$H_{i j}(\mathrm{eV})$ & $-1.31 \times 10^{-2}$ & $-2.47 \times 10^{-2}$ \\
No of $H_{i j}(i<j)$ & 4095 & 2162 \\
Matrix dimension & $91 \times 91$ & \\
$D(\mathrm{eV})$ & & 0.31 \\
$\frac{V}{D}$ & & 2.357 \\
\hline
\end{tabular}

For both coupling schemes, $\sqrt{\overline{H_{i j}^{2}}}$ for even Sm IX is $\approx 0.53 \mathrm{eV}$ and for odd Sm IX it is $\approx 0.31 \mathrm{eV}$, when the whole matrix is considered. When $H_{i j} \neq 0$ only is considered, $\sqrt{\overline{H_{i j}^{2}}}$ for even Sm IX is $\approx 0.73$ or $\approx 0.93 \mathrm{eV}$ and for odd Sm IX it is $\approx 0.4$ or $\approx 0.68 \mathrm{eV}$, depending on the coupling scheme (see tables 2 and 3). In both even and odd Sm IX, the ratio $V / D$
Table 3. Statistical characteristics of the off-diagonal Hamiltonian matrix elements of odd Sm IX in $l s$ and $j j$ coupling.

\begin{tabular}{lll}
\hline & Whole matrix & $H_{i j} \neq 0$ only \\
\hline Sm IX odd $l s$ & & \\
$\overline{H_{i j}^{2}}\left(\mathrm{eV}^{2}\right)$ & 0.095 & 0.468 \\
$\sqrt{\overline{H_{i j}^{2}}}(\mathrm{eV})$ & 0.309 & 0.684 \\
$H_{i j}(\mathrm{eV})$ & $-6.08 \times 10^{-3}$ & $-2.98 \times 10^{-2}$ \\
No of $H_{i j}(i<j)$ & 21945 & 4474 \\
Matrix dimension & $210 \times 210$ & \\
$D(\mathrm{eV})$ & & 0.101 \\
$\frac{V}{D}$ & & 6.79 \\
$\mathrm{Sm} \mathrm{IX} \mathrm{odd} j j$ & & \\
$\overline{H_{i j}^{2}}(\mathrm{eV})$ & 0.094 & 0.158 \\
$\sqrt{\overline{H_{i j}^{2}}}(\mathrm{eV})$ & 0.3064 & 0.397 \\
$H_{i j}(\mathrm{eV})$ & $-3.42 \times 10^{-3}$ & $-5.75 \times 10^{-3}$ \\
No of $H_{i j}(i<j)$ & 21945 & 13049 \\
Matrix dimension & $210 \times 210$ & 0.1 \\
$D(\mathrm{eV})$ & & 3.98 \\
$\frac{V}{D}$ & & \\
\hline
\end{tabular}

(where $V=\sqrt{\overline{H_{i j}^{2}}}, H_{i j} \neq 0$ only; $D$ is the mean level spacing of the unperturbed $H_{0}\left(H_{i j}\right)$ energy levels) for $l s$ coupling is approximately twice that in the $j j$ coupling scheme; i.e. this strongly suggests basis-dependent results for values of $V$. For even Sm IX, $D=0.31 \mathrm{eV}$ while for odd Sm IX, $D=0.1 \mathrm{eV}$. Since the ratio $V / D \gg 1$, this suggests the possibility of chaotic mixing of states. 

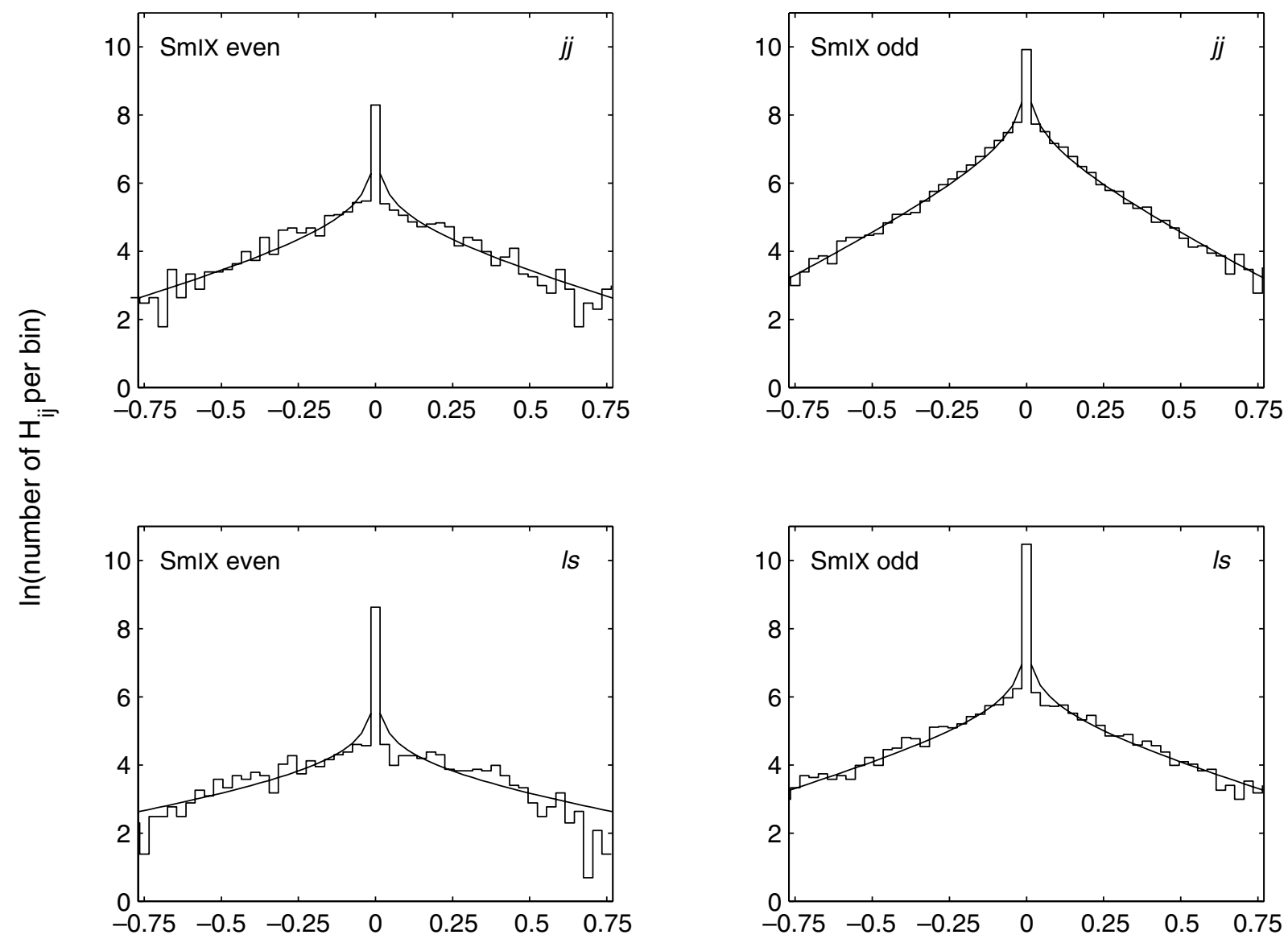

$\mathrm{H}_{\mathrm{ij}}(\mathrm{eV})$

Figure 2. Distribution of off-diagonal elements of the Sm IX Hamiltonian matrix. The solid curve is given by equation (3).

The distributions of the off-diagonal $H_{i j}$ elements are shown in figure 2 . The even and odd matrices are remarkably similar apart from the small vertical shift due to the different size of the matrices. All histograms show very large numbers of small matrix elements as expected, since the matrix elements between distant configurations become very small when the configurations differ by states of at least two electrons. For the off-diagonal matrix elements, $H_{i j}>$ $0 \mathrm{eV}$, the distribution can be approximated by the following exponential formula [9],

$$
\frac{\Delta N}{\Delta H} \propto\left|H_{i j}\right|^{-1 / 2} \exp \left(\frac{-\left|H_{i j}\right|}{V}\right)
$$

as shown by the logarithmic plots in figure 2. The off-diagonal $H_{i j}$ elements were curve fitted using the following function

$$
F(x, \alpha, \beta)=\alpha\left(|x|^{-1 / 2}\right) \exp \left(\frac{-|x|}{\beta}\right)
$$

where $\alpha$ and $\beta$ for the least-squares fitting of the nonzero $H_{i j}$ are given in table 4 . The values of $\beta$ are not consistent with the corresponding values of $\sqrt{\overline{H_{i j}^{2}}},\left(H_{i j} \neq 0\right.$ only) ( $\beta$ on average is $30 \%$ lower).
Table 4. Various curve fitting parameters for even and odd Sm IX.

\begin{tabular}{llllll}
\hline & \multicolumn{3}{c}{ Sm IX even } & \multicolumn{2}{c}{ Sm IX odd } \\
\cline { 2 - 3 } \cline { 5 - 6 } & $l s$ & $j j$ & & $l s$ & $j j$ \\
\hline$\alpha$ & 31.12 & 68.08 & & 132.58 & 560.28 \\
$\beta$ & 0.82 & 0.45 & & 0.44 & 0.24 \\
$k$ & 0.005 & 0.007 & & 0.001 & 0.002 \\
$S_{0}$ & 0.51 & 0.75 & & 0.31 & 0.74 \\
$H_{0}$ & 1.63 & 1.37 & & 0.94 & 0.67 \\
$b$ & 20.79 & 18.60 & & 74.68 & 54.62 \\
& $0.23 N$ & $0.2 N$ & & $0.36 N$ & $0.26 N$ \\
$\overline{\Delta E}(\mathrm{eV})$ & 4.54 & 4.67 & & 3.95 & 4.05 \\
$\overline{\Delta E} / D$ & 14.66 & 15.06 & 39.2 & 40.63 \\
\hline
\end{tabular}

\subsection{Dependence of $H_{i j}$ on their distances} from the diagonal

Figure 3 shows that the matrix elements $H_{i j}$ decrease in magnitude as the distance from the diagonal $\Delta=|i-j|$ [14] increases. The matrix elements appear more dispersed in the $l s$ coupling scheme than in the $j j$ coupling scheme for both even and odd Sm IX with large off-diagonal elements in both even and odd Sm IX and in both coupling schemes.

\subsection{Sparsity of $H_{i j}$}

The sparsity $S$ of the Hamiltonian matrix defined for $|i-j|=$ $\Delta$ as the ratio of the number of nonzero matrix elements to the 

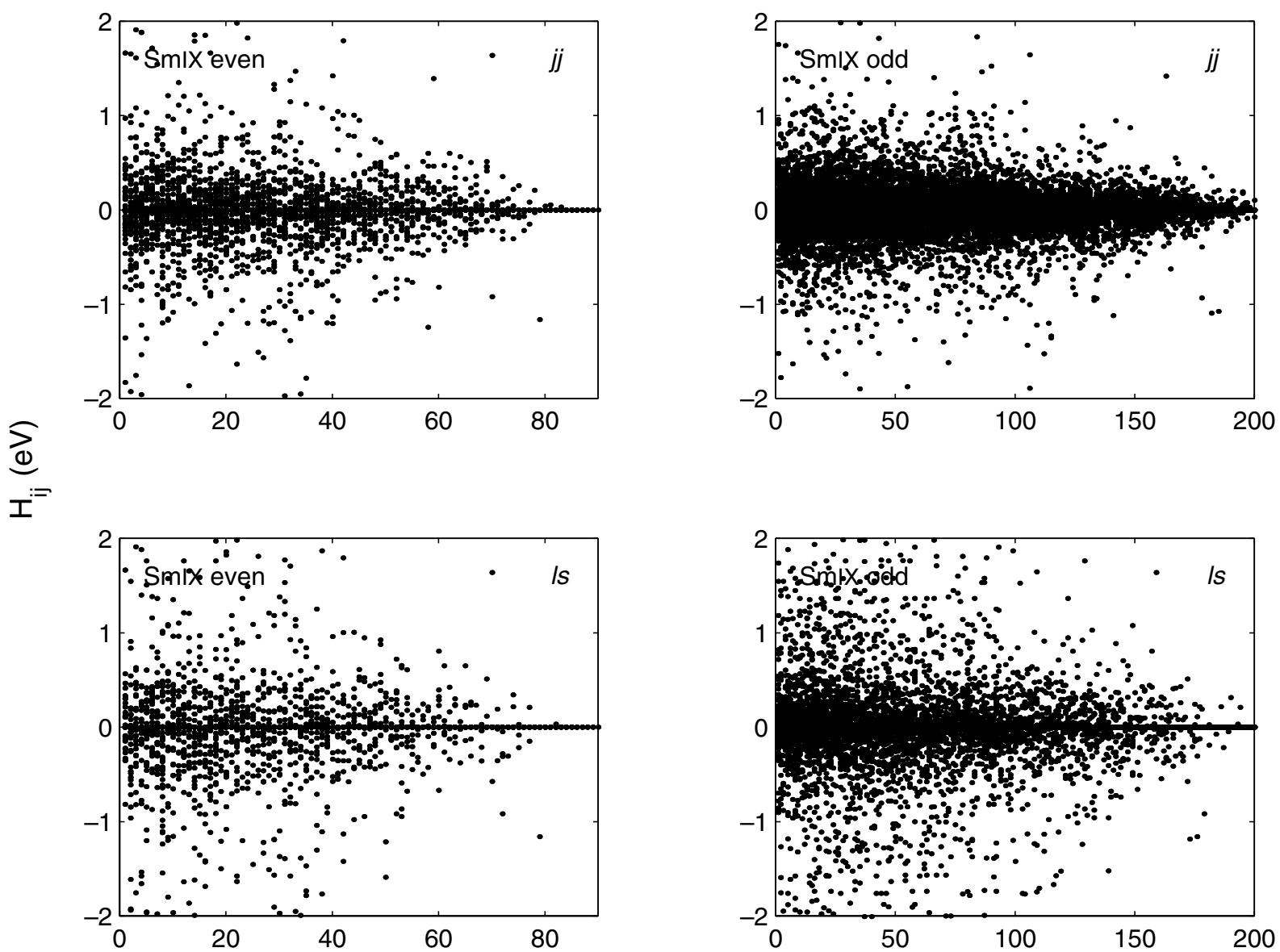

$|i-j|$

Figure 3. Matrix elements $H_{i j}$ of Sm IX as a function of the distance to the main diagonal $|i-j|$.

total number of matrix elements [12],

$$
S=\frac{\text { number of }\left|H_{i j}\right| \neq 0}{\text { number of all } H_{i j}} \quad|i-j| \text { fixed, }
$$

is presented in figure 4. According to this definition the sparsity is 1 for full random matrices. In the $j j$ coupling scheme, the sparsity is approximately constant for $|i-j| \leqslant$ $N / 2$, where $N$ is the matrix dimension, and then there is a definite decrease in the sparsity. For the $l s$ coupling scheme, the sparsity is approximately constant for $|i-j| \leqslant N / 4$, and then there is a definite decrease in the sparsity. The sparsity is greater in the $j j$ coupling scheme, and at small $|i-j|$ closer to the value for full random matrices, than in the $l s$ coupling scheme. The sparsity was fitted according to [12]

$$
S(\Delta)=S_{o}-k \Delta,
$$

where parameters of the fit, $S_{0}$ and $k$, are also given in table 4.

\subsection{Number bandwidth $b$}

The number bandwidth $b$ was estimated by fitting the meansquared matrix elements to [12]

$$
\left\langle H_{i j}^{2}\right\rangle_{|i-j|=\Delta}=H_{0}^{2} \exp \left(-\frac{\Delta}{b}\right)
$$

where $\left\langle H_{i j}^{2}\right\rangle_{|i-j|=\Delta}$ is the average of the squared nonzero matrix elements at a given distance $\Delta$ from the diagonal. The values of $b$ compared with the matrix dimension $N$ are given in table 4 . In all cases, $b$ was found to be much smaller than $N$ showing the banded nature of the matrices.

\subsection{Energy bandwidths $\Delta E_{i}$}

The energy bandwidths $\Delta E_{i}$ were calculated according to $[9,12]$

$$
\left(\Delta E_{i}\right)^{2}=\frac{\sum_{j}\left(H_{i i}-H_{j j}\right)^{2}\left|H_{i j}\right|^{2}}{\sum_{j \neq i}\left|H_{i j}\right|^{2}} .
$$

The overall mean energy bandwidths $\overline{\Delta E}$ as given in table 4 are seen to be fairly constant. This energy bandwidth is a more physical characteristic of the Hamiltonian matrix, since it refers to the energy interval within which the perturbation strongly mixes the basis states. The number bandwidth $(b=$ $\overline{\Delta E} / D)$ is seen to be $\approx 15$ for even Sm IX and $\approx 40$ for odd Sm IX reflecting the greater energy spread in the latter (see table 1).

\subsection{Discussion I}

As has been the case for the rare-earth elements studied so far, the Hamiltonian matrices of even and odd Sm IX in 

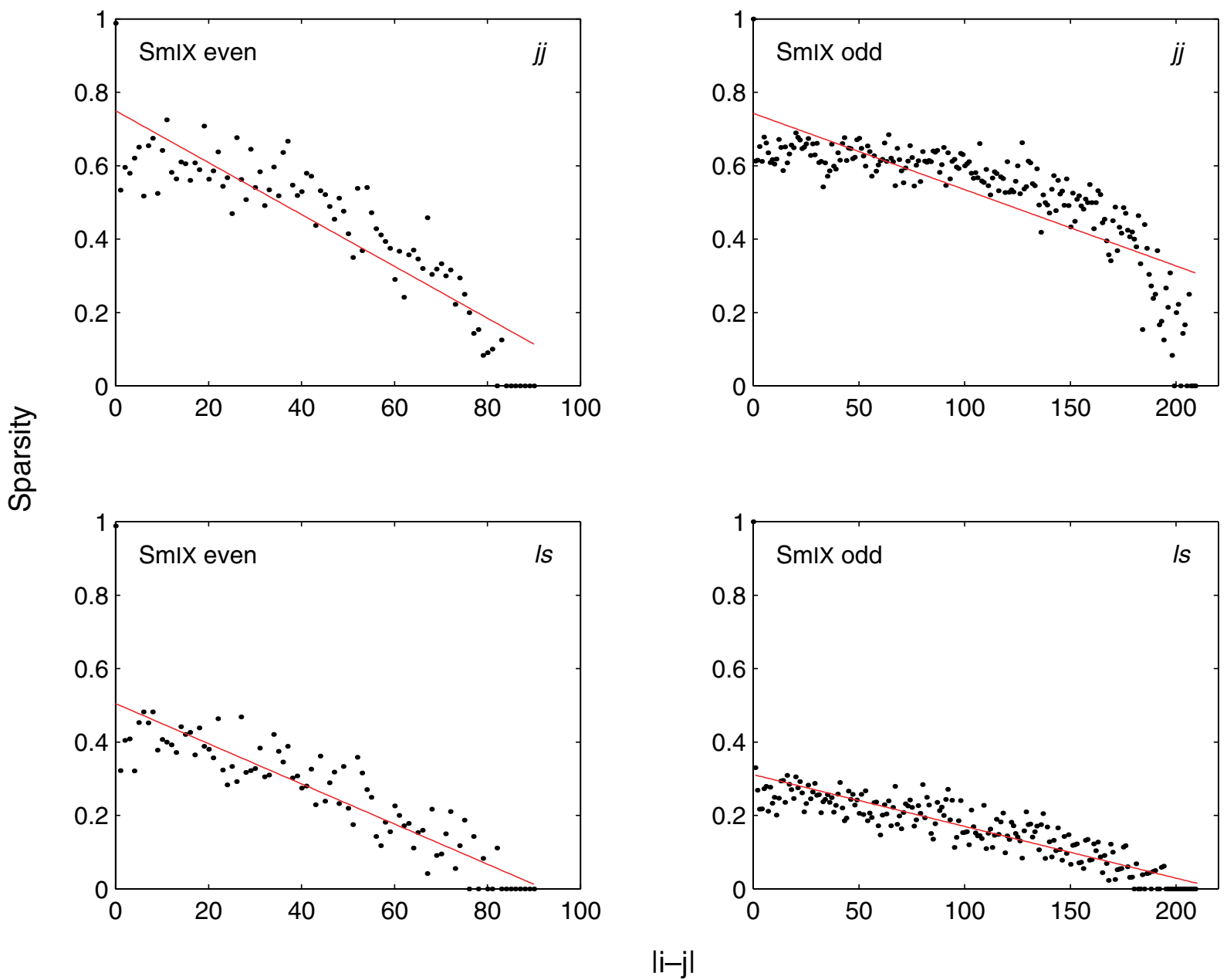

Figure 4. Sparsity $S$ of the Sm IX Hamiltonian matrix.

$l s$ and $j j$ coupling schemes can be characterized as sparse band matrices with a leading diagonal. However, BRM cannot account for the existence of some nonzero off-diagonal matrix elements due to the strong coupling of distant basis states by the perturbation.

\section{Structure of the eigenstates and the basis states}

Figures 5 and 6 show examples of energy eigenstates $|j\rangle$, with components $C_{i}$ in a basis $|i\rangle$, and basis states, with components $C_{j}$ in a basis $|j\rangle$, for odd Sm IX. The eigenstates are distributed among a large number of component basis states and form compound atomic states. This is also true for the basis states as a function of $|j\rangle$.

The states are well localized on the energy scale, i.e., the components contributing to a particular eigenstate are mainly confined within an energy range $\sim \pm 10 \mathrm{eV}$ around the centroid energy. This behaviour agrees with the existence of a characteristic spreading width $\Gamma$ [9], which determines the energy range within which the basis states are strongly mixed by the perturbation and hence the range of eigenvalues to which a given basis state noticeably contributes. A relative shift of the large components envelope following the increase of the eigenstate energy can be observed. This means the localization of components around the eigenstate they represent. Due to the finite size of the matrix, the eigenstates are more localized in the middle of the spectrum than at the upper and lower bounds. The energy centres of the eigenstates tend to increase as the energy of the eigenstates increases. This is also true of the basis states as a function of $|j\rangle$.

\subsection{EFs and LDOS}

The shape of an EF, given by the dependence of $W_{i}^{j}$ on $i$ for fixed values of $j\left(W_{i}^{j}=\left|C_{i}^{j}\right|^{2}\right.$ ( $C$ is the amplitude) where the subscripts refer to the basis states and the superscripts correspond to the compound eigenstates), is defined as [32]

$$
w_{j}\left(E^{0}\right)=\sum_{i}\left|C_{i j}\right|^{2} \delta\left(E^{0}-E_{i}^{0}\right)
$$

in the unperturbed energy basis. The local density of states (LDOS) is given by the dependence of $W_{i}^{j}$ on $j$ for fixed values of $i$. The spectral density of states (LDOS) for an unperturbed state $|i\rangle$ is defined as [32]

$$
w_{i}(E)=\sum_{j}\left|C_{i j}\right|^{2} \delta\left(E-E_{j}\right)
$$

where $E_{j}$ is the eigenenergy of the perturbed eigenstate $|j\rangle$ and $C_{i j}=\langle i \mid j\rangle$. The mean-squared components $\left\langle\left|C_{i j}\right|^{2}\right\rangle$ were calculated and in order to suppress fluctuations, window averaged over \pm 5 . Before averaging, $w_{i}(E)$ and $w_{j}(E)$ are 

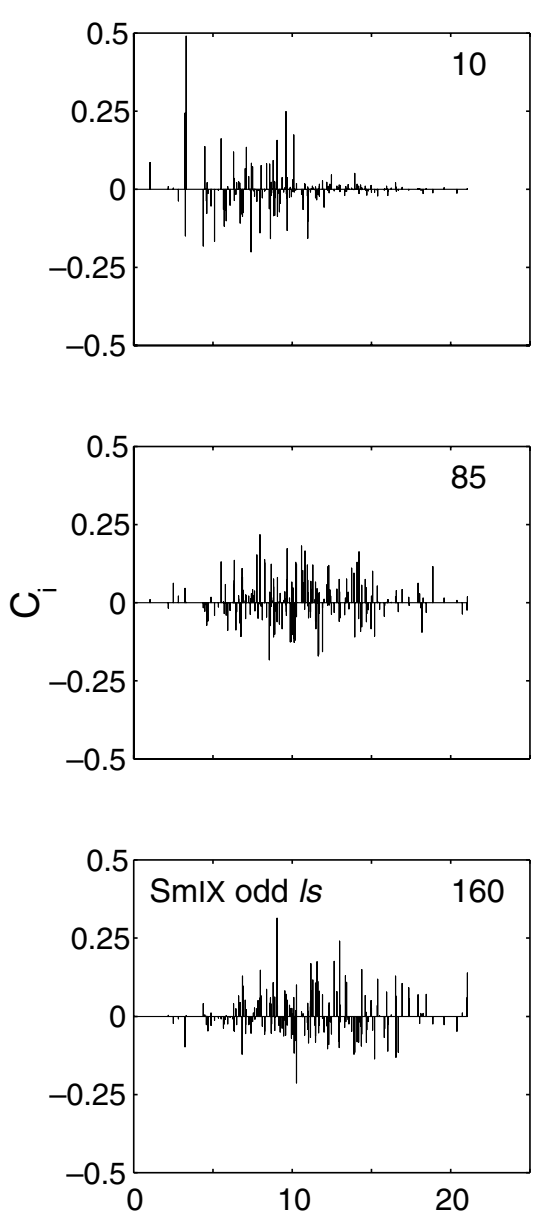
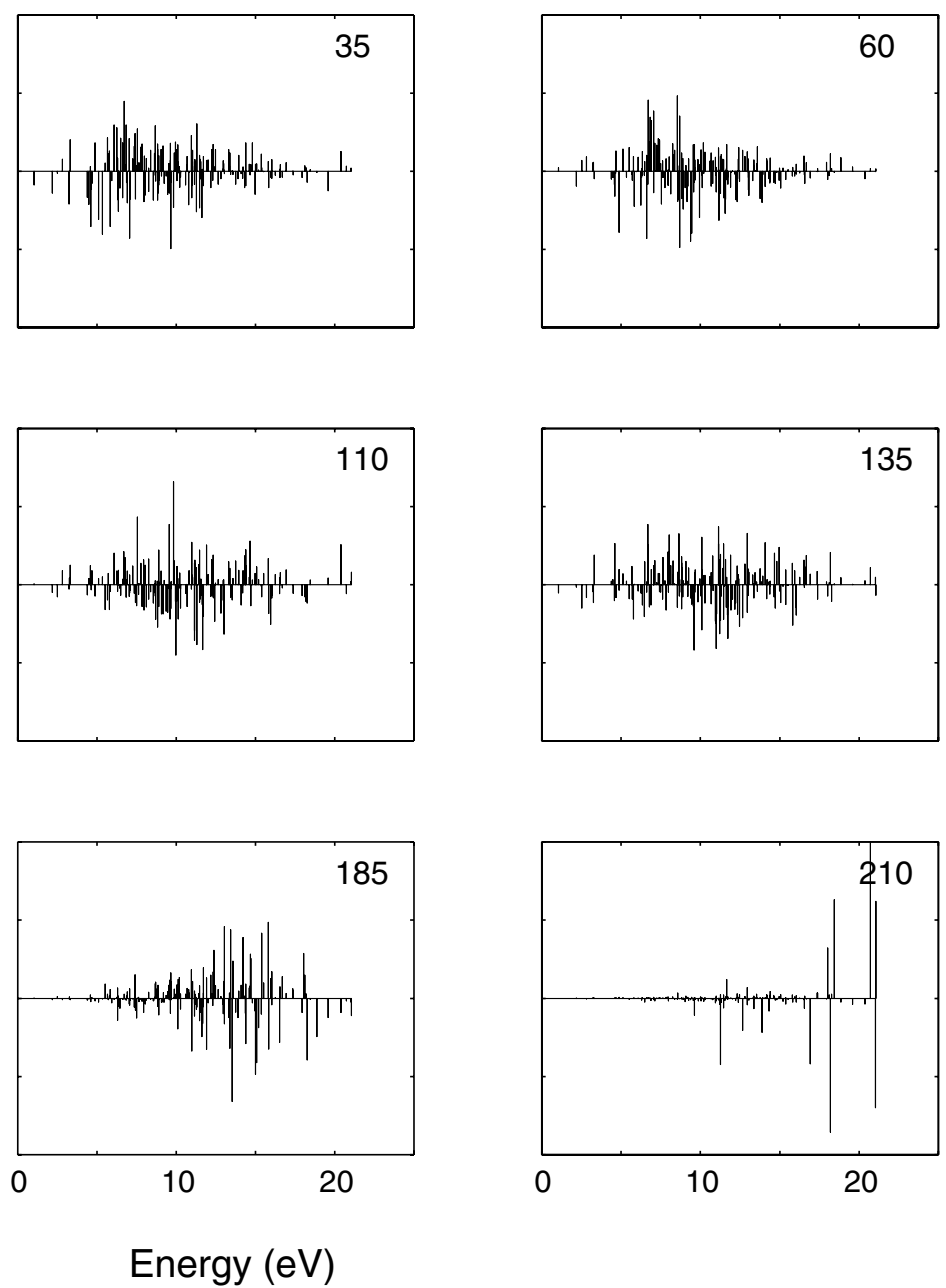

Figure 5. Various eigenstates of odd Sm IX in $l s$ coupling. The eigenstate components $C_{i}$ are shown as a function of the basis state energies.

expressed with respect to their centroids. For the EFs the centroid of $w_{j}\left(E^{o}\right), e_{j}$, is defined by [32]

$$
e_{j}=\sum_{i} E_{i}^{0}\left|C_{i j}\right|^{2}
$$

and $w_{j}$ can be expressed as a function of the shift $\left(E^{0}-e_{j}\right)$. For the LDOS, the centroid of $w_{i}(E)$ is [32]

$$
E_{i}^{0}=\sum_{j} E_{j}\left|C_{i j}\right|^{2},
$$

so the LDOS can be expressed as $w_{i}\left(E-E_{i}^{0}\right)$.

The window-averaged EFs and LDOS, plotted in figures 7 and 8 show the presence of localization. A banded structure is clearly seen in the shape of the EFs. This banded structure is also evident in the LDOS although the centre of the band is seen to be shifted to higher $i$, as $i$ increases. The localization of the eigenstates as seen in the EFs (and correspondingly in the LDOS) implies that a perturbation mixes the basis states locally and the components of a given eigenstate rapidly vanish as one moves away from the 'centre' of the eigenstate. In general, the LDOS and EFs are skewed at low and high states, but are more symmetric in the middle of the spectra. This is partly due to the low states being bounded from below, i.e. in a finite basis the very low and very high basis states cannot achieve the same degree of coupling — on an average they are only coupled to $\approx b$ basis states, unlike the 'middle' states, which can couple to $\approx 2 b$ basis states. This range of coupling (i.e. the bandedness of the Hamiltonian matrix) results in the localization of the EFs and the $\operatorname{LDOS}[9,17]$.

The LDOS and EFs had the following functions fitted to them [9]:

$$
w\left(E_{i} ; E+\Delta E, \Gamma, N\right)=N^{-1} f(\varepsilon)
$$

in the energy region $-15 \rightarrow 15 \mathrm{eV}$, where the shape function $f(\varepsilon)$ has the forms for various distributions as detailed in [9]. This follows from the work of Wigner [1, 2], Fyodorov et al [31] and Wang et al [32]. It was shown that for BRMs when the coupling between the matrix elements is constant, the LDOS has the form of the Breit-Wigner law (similar to the fitted Lorentzian) [33]. For larger perturbations, the form of the LDOS can be described by a Gaussian [31]. Typical window-averaged EFs and LDOS with the least-squares fitted functions are shown in figures 9 and 10. The 33rd and 108th states (chosen close to the centre of the EFs and LDOS distributions) are shown for even and odd Sm IX respectively. These states are shown for both coupling schemes. The $\left\langle\left|C_{i j}\right|^{2}\right\rangle$ are larger in the 'middle' of the spectra, indicating that the $C_{i j}$ are more localized over the basis states/eigenstates 

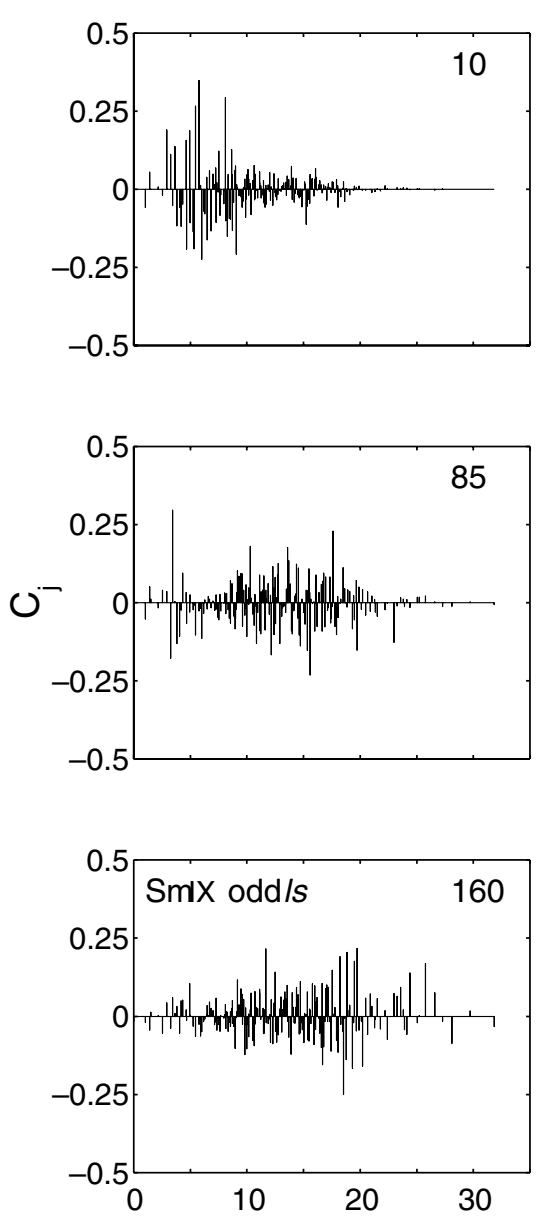
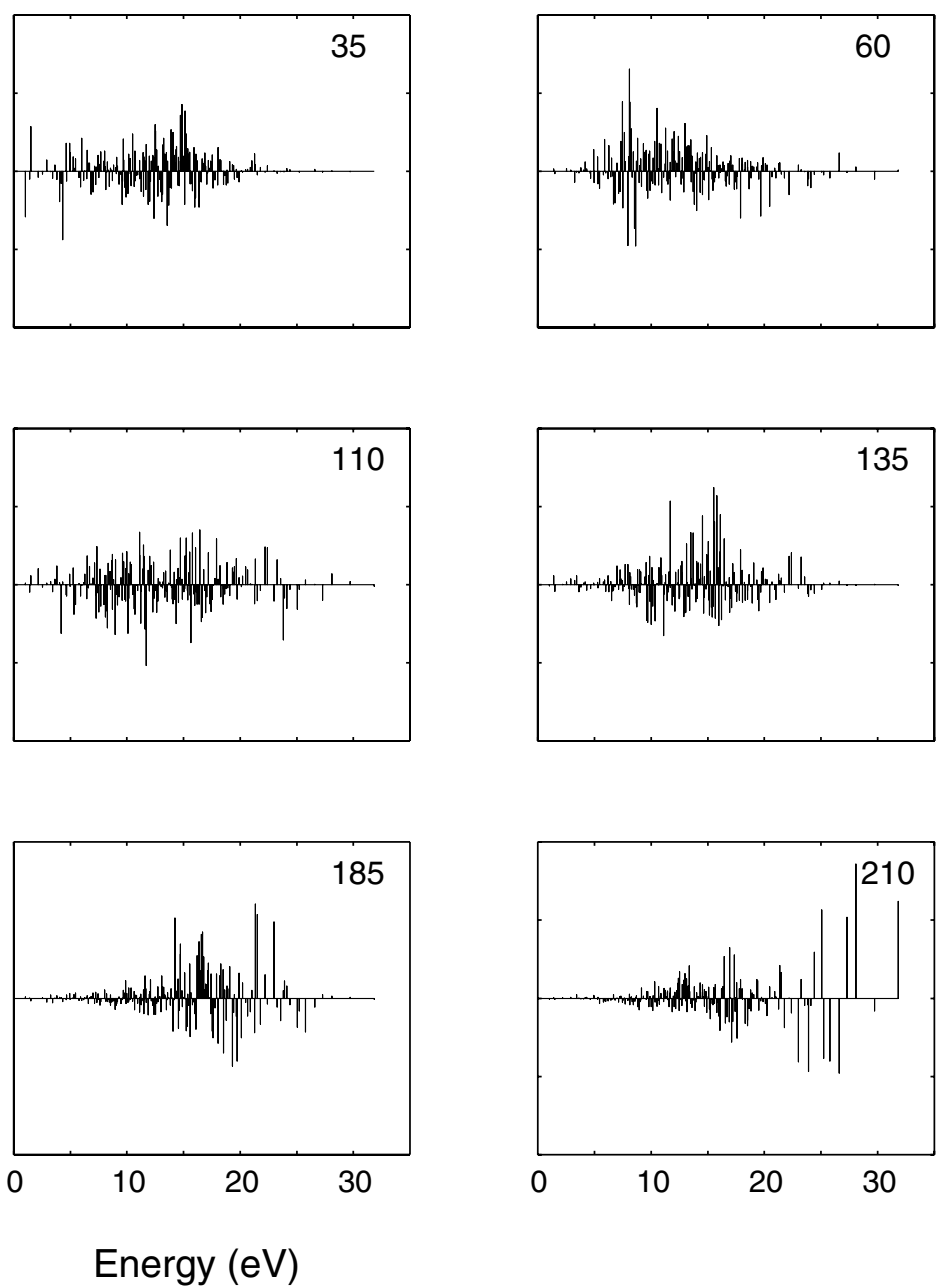

Figure 6. Various basis states of odd Sm IX in $l s$ coupling. The basis state components $C_{j}$ are shown as a function of the eigenstate energies.

than the high or low spectral regions. The window-averaged LDOS are broader in the central region than the corresponding EFs.

The sum of the squares of the residuals (SSR) were calculated using a nonlinear least-squares algorithm as an indication of the goodness of the fits. For the EFs it was found that the Lorentzian had the lowest SSR whereas the Gaussian fits had the greatest SSR. For the LDOS, it was found that the Gaussian and interpolation exponential had the lowest SSR and the Lorentzian had the largest SSR.

\subsection{Number of principal components $N$}

The number of principal components $N$ indicates the number of components that contributes significantly to a given eigenstate (basis state). It was calculated for various $f(\varepsilon)$ distributions and it was found that $N$ reaches a maximum of approximately 60 for even Sm IX, and 150 for odd Sm IX, which is $\sim$ two-third of the number of available states for both the EFs and LDOS. The maximum value of $N$ extends over a similar energy range $(\sim 5 \mathrm{eV})$ for even Sm IX and odd Sm IX in both the $l s$ coupling scheme and the $j j$ coupling scheme and for both EFs and LDOS. The maxima $N$ values occur in the central energy regions. The value of $N$ for the corresponding LDOS and EFs for each parity and coupling scheme are very similar in shape and magnitude.

\subsection{The spreading width $\Gamma$}

The spreading width $\Gamma$ for the EFs indicates the energy region over which substantial mixing between basis states occurs around the unperturbed centroid basis state. $\Gamma$ for the LDOS indicates the width of the energy region over which the initial unperturbed basis state becomes 'spread'. It was found to range between $\sim 5$ and $10 \mathrm{eV}$ for the Lorentzian and interpolation exponential, and between $\sim 10$ and $20 \mathrm{eV}$ for the Lorentzian squared, in both the EFs and LDOS and for both coupling schemes. Similar $\Gamma$ 's for the EFs and LDOS indicate that the two-body interaction in the Hamiltonian is relatively small. Fermi's golden rule gives for the BW function

$$
\Gamma_{B W}=2 \pi \frac{\overline{V^{2}}}{D}
$$

where $V=\langle a|H| b\rangle, a \neq b$ (i.e. off-diagonal elements) and the 'bar' indicates the mean. Using the $\overline{H_{i j}^{2}}$ values of table 4 (the whole matrix values), the $\Gamma_{B W}$ were calculated and are tabulated in table 5 .

There is no substantial difference between the $\Gamma_{B W}$ values of the corresponding coupling schemes. These values 

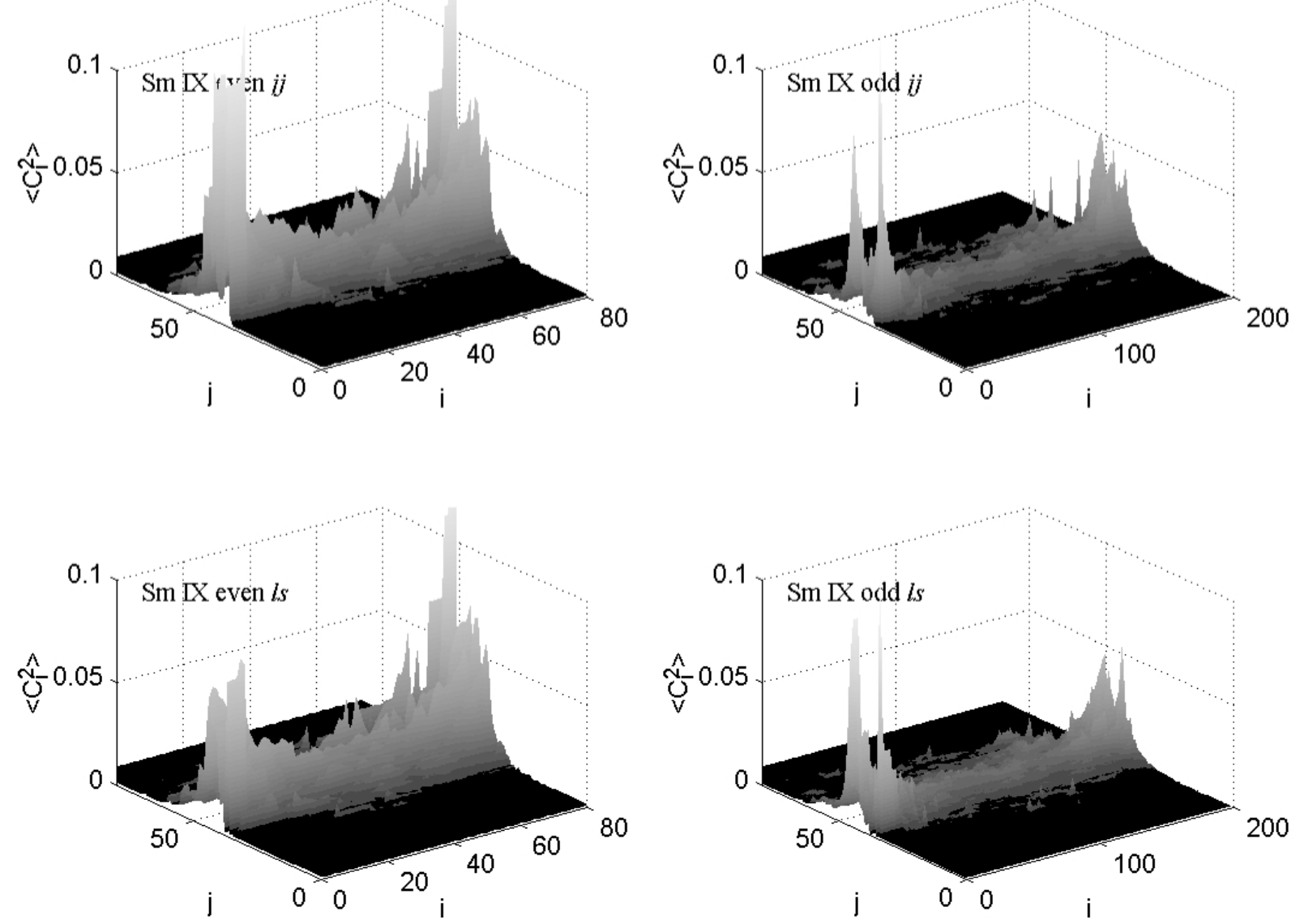

Figure 7. Window-averaged EFs for Sm IX.
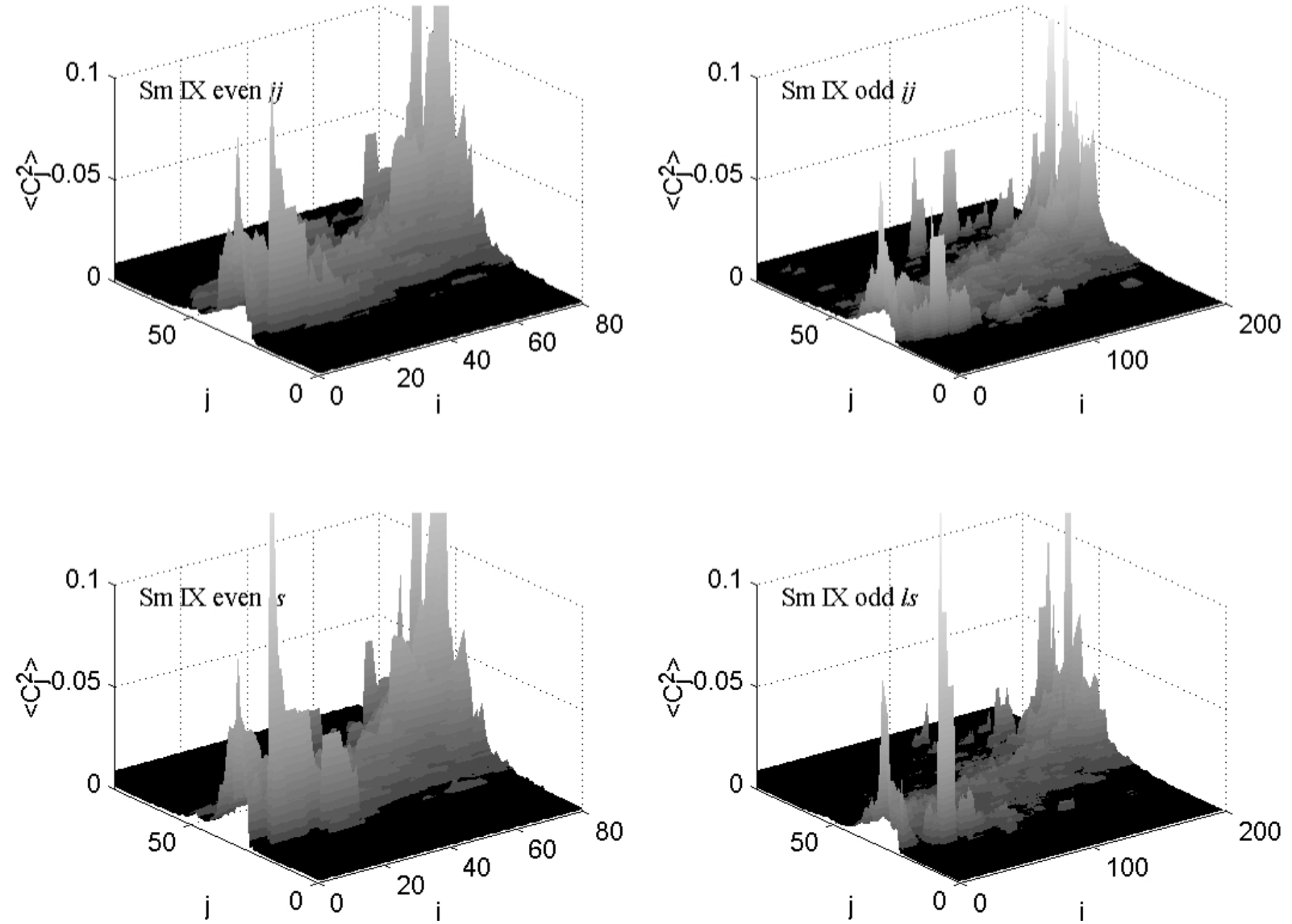

Figure 8. Window-averaged LDOS for Sm IX. 

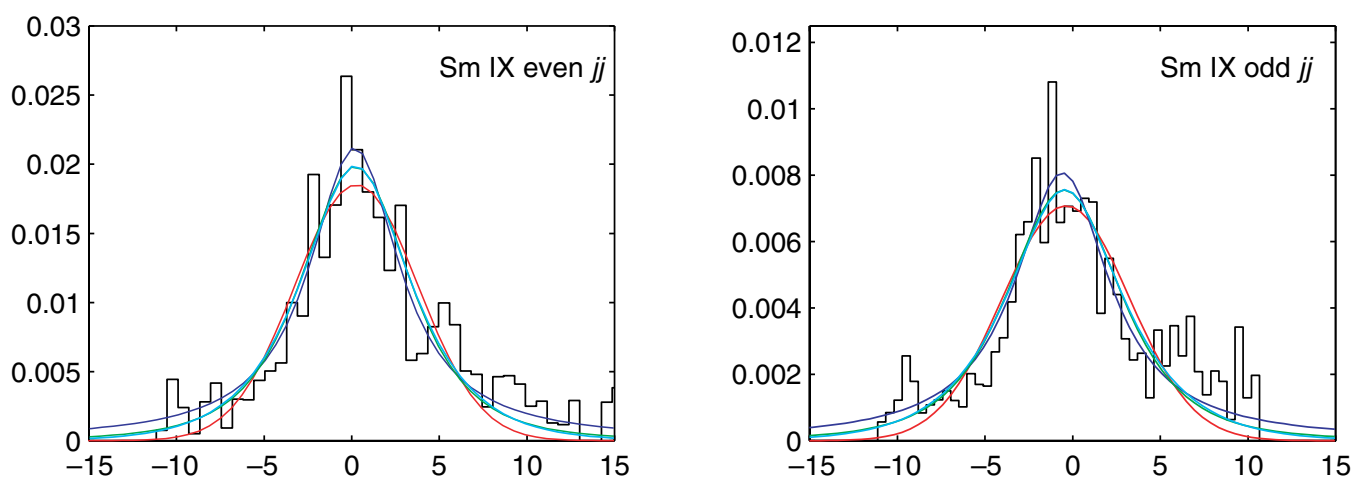

$<C_{i}^{2}>$
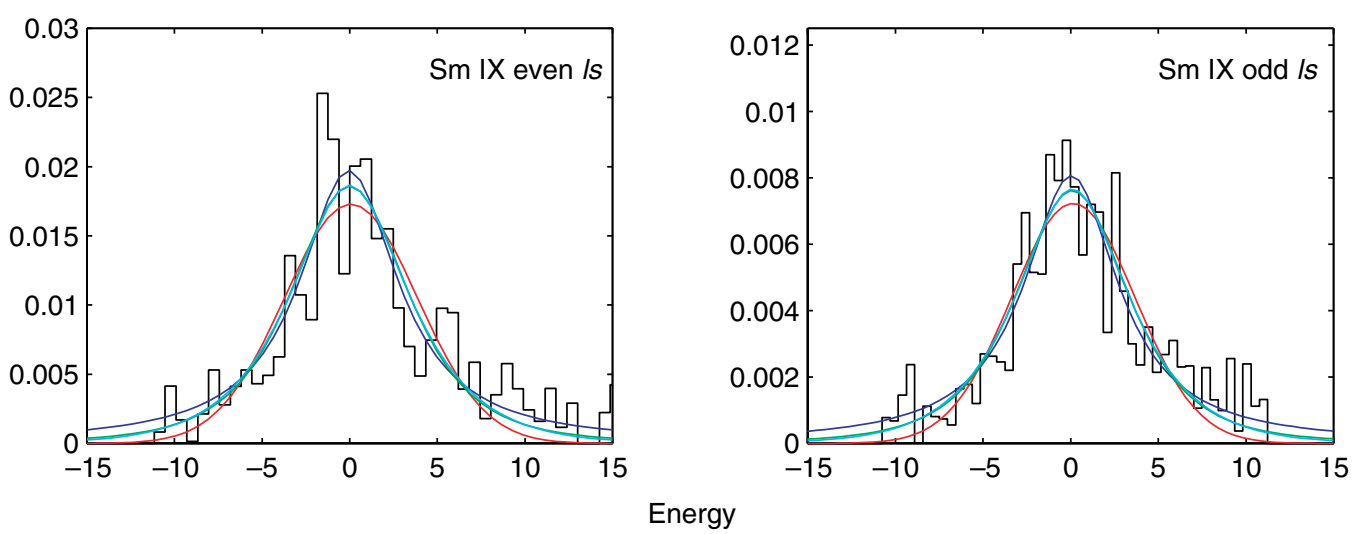

Figure 9. Selected window-averaged EFs for Sm IX and least-squares fitting to Gaussian (red), Lorentzian (blue), squared Lorentzian (green) and interpolation exponential (cyan).
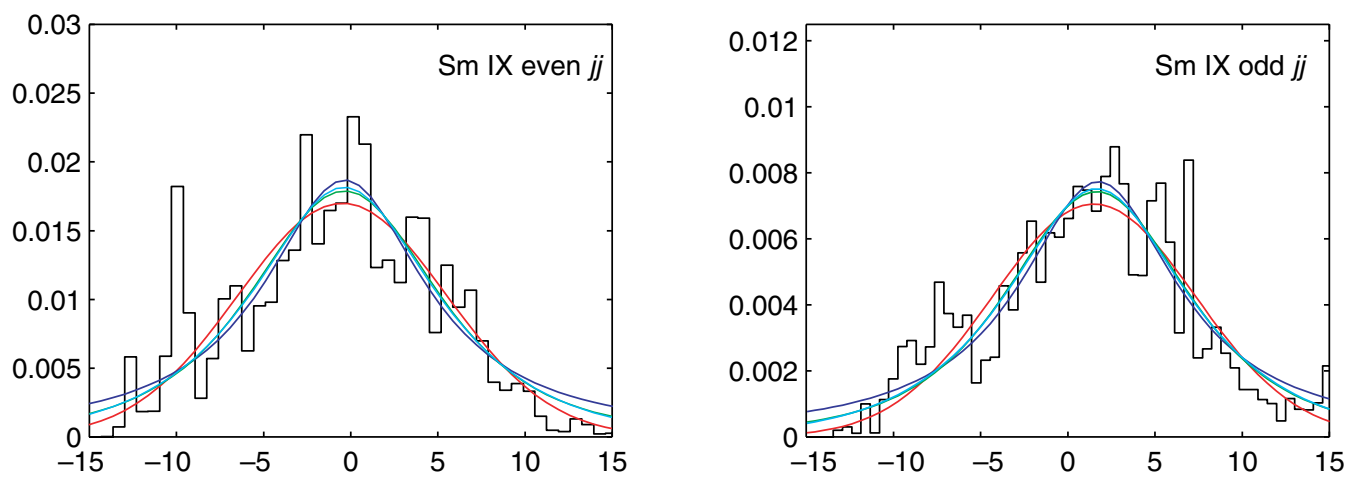

$\left\langle\mathrm{C}_{\mathrm{j}}^{2}>\right.$
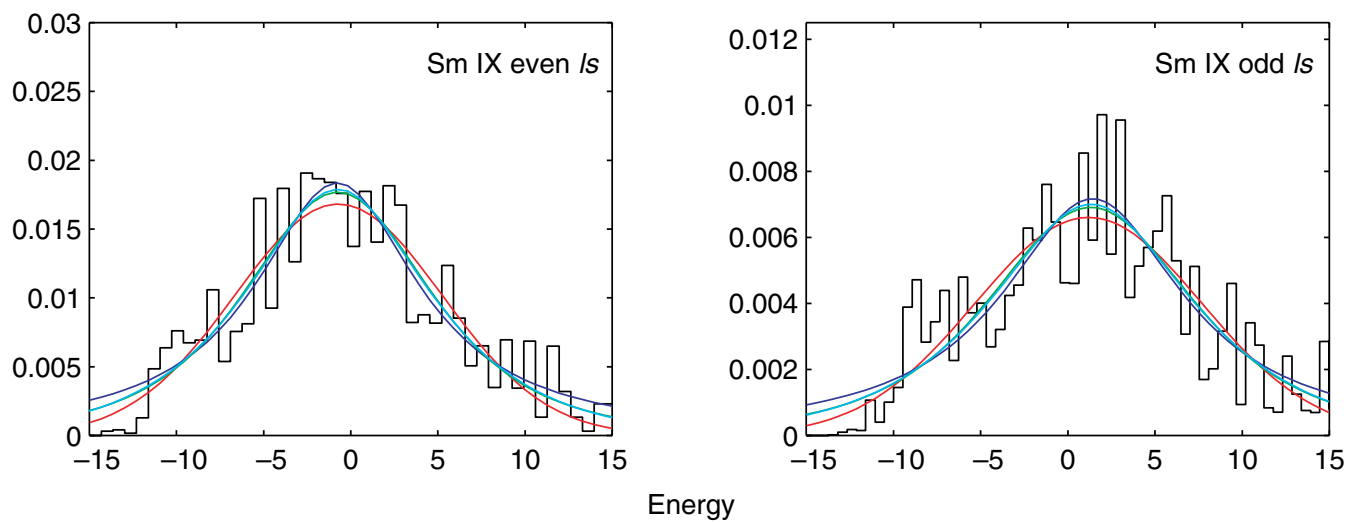

Figure 10. Selected window-averaged LDOS for Sm IX and least-squares fitting to Gaussian (red), Lorentzian (blue), squared Lorentzian (green) and interpolation exponential (cyan). 
Table 5. $\Gamma_{B W}$ for even and odd Sm IX, calculated using Fermi's golden rule.

\begin{tabular}{llllll}
\hline & \multicolumn{3}{l}{ Sm IX even } & \multicolumn{2}{l}{ Sm IX odd } \\
\cline { 2 - 3 } \cline { 6 - 6 } & $l s$ & $j j$ & & $l s$ & $j j$ \\
\hline$\Gamma_{B W}=2 \pi \frac{\bar{V}^{2}}{D}$ & 5.75 & 5.71 & & 5.91 & 5.90 \\
\hline
\end{tabular}

Table 6. Density of states, $\rho(E)$, for the Lorentzian, squared Lorentzian and interpolation exponential distributions.

\begin{tabular}{ll}
\hline$\rho(E)=\frac{2 N}{\pi \Gamma}$ & Lorentzian \\
$\rho(E)=\frac{4 N}{\pi \Gamma}$ & Squared Lorentzian \\
$\rho(E)=\frac{N}{1.636 \Gamma}$ & Interpolation exponential \\
\hline
\end{tabular}

are consistent with the $\Gamma$ 's calculated via the Lorentzian and interpolation exponential curve fits. The energy shifts $\Delta E$ for the EFs were found to vary from $\sim-1 \rightarrow 1 \mathrm{eV}$ and $-2 \rightarrow$ $2 \mathrm{eV}$ for even and odd Sm IX respectively for both coupling schemes. The energy shifts $\Delta E$ for the LDOS were found to increase from $-5 \rightarrow 10 \mathrm{eV}$ for even and odd Sm IX and for both coupling schemes. This is expected since the centre of the LDOS increases with $i$ as shown in figure 8. If the width of the perturbed spectrum is of the same order as the unperturbed one, it is expected that both shapes (LDOS and EFs) are very close to each other $[17,30]$.

\subsection{Density of states}

Table 6 summarizes the various densities of states $\rho(E)$ corresponding to different distributions. Figure 11 compares the calculated density of states $\rho$ for Sm IX with the value of $\rho$ for the Lorentzian distribution. It was found that the $\rho$ given by the Lorentzian distribution appears to be a better approximation for the density of states than the squared Lorentzian and the interpolation exponential.

\subsection{Complexity of wavefunction components}

The information entropy, $S[4,34-36]$, measures the degree of complexity of individual wavefunction components $C_{i j}$. It is defined for a given normalized wavefunction $|j\rangle$ and expanded with the aid of a given basis $|i\rangle$, in terms of the weights of the components

$$
S^{j}=-\sum_{i} W_{i}^{j} \ln \left(W_{i}^{j}\right),
$$

where $W_{i}^{j}=\left(C_{i}^{j}\right)^{2}$ and $C_{i}^{j}=$ normalized amplitude. In a given energy range, $E \approx E_{j}$, the distribution of components $C_{i}^{j}$ is similar to a Gaussian one but with the local width $\overline{\left(C_{i}^{j}\right)^{2}}=1 / N_{j}$ :

$$
P^{j}\left(C^{j}\right)=\sqrt{\frac{N_{j}}{2 \pi}} \exp \left[-\frac{N_{j}}{2}\left(C^{j}\right)^{2}\right],
$$

where $N_{j}$ is the number of principle components [16]. The entropy $S^{j}$, or the corresponding length in Hilbert space $l_{s}^{j}=\exp \left(S^{j}\right)$, characterizes the degree of delocalization of a given $\mathrm{EF}|j\rangle$ with respect to an original basis. The deviation of $l_{s}^{j}$ from the GOE limit $0.482 N$ indicates the incomplete mixing of basis states [16]. Figure 12 shows the calculated $\exp \left(s^{\alpha}\right)$, the information length, where $\alpha$ represents either the eigenvectors $|j\rangle$ or the basis vectors $|i\rangle$.

In the most chaotic part (the centre) of the spectrum, the information entropy comes very close to reaching the GOE value, $N_{j}=N$. The maximum $l_{s}^{j}$ value is $\sim 90$ for odd Sm IX and $\sim 35$ for even Sm IX for both coupling schemes. This indicates mixing of the basis states at the centre of the spectrum. However, there is incomplete mixing over the whole spectrum. A typical pattern of the regular bell-shape behaviour of information entropy $S$ is formed due to the CI.

\subsection{Localization length}

The size of the basis which eigenstates occupy is defined via the entropy localization length, $l_{h}$, defined as

$$
l_{h}=N \exp \left(\langle H\rangle-H_{0}\right),
$$

where $\langle H\rangle$ is the mean entropy of eigenstates

$$
\langle H\rangle=-\frac{1}{M} \sum_{n=1}^{M} \sum_{i=1}^{N} W_{i}^{n} \ln \left(W_{i}^{n}\right),
$$

and $H_{0}$ is the normalization constant, which is equal approximately to 2.078 in the pure Gaussian fluctuations of $C_{i}$ [4]. $M$ is the number of eigenstates which are taken for the average ( $M=1$ in this work). The dependence of the entropy localization length $l_{h}$ on the energy is shown in figures 13 and 14.

It can be seen that $l_{h}$ reaches approximately $90 \%$ of the maximum of the GOE values of $N$ (the matrix size) for both $l s$ and $j j$ couplings. Also there is no significant difference between the $l_{h}$ of different coupling schemes. The circles indicate $3.5 N P C$ (where $N P C$ is the effective number of principal components [16]) compared to $4 N P C$ predicted in [9] for the Lorentzian distribution.

\subsection{Discussion II}

It has been shown that the excited states of the rare-earth ion Sm IX have possible chaotic structure. These states are similar to the compound resonances in heavy nuclei. They are formed as superpositions of large numbers of single-electron basis states. The mixing of the basis states determines the strength of the perturbation (coupling due to $\mathrm{CI}$ ) and the level spacing of the spectrum. In general, the EFs are Lorentzian in shape while the LDOS are Gaussian in shape showing localization in energy with a spreading width $\Gamma$. The degree of complexity of wavefunctions can be measured by the information entropy and the localization length of the various wavefunctions was found to be very close to the prediction of RMT close to the centre of the distribution.

\section{Energy level statistics}

Diagonalization of the Hamiltonian gives a set of energy eigenvalues that are analysed and compared with the predications of RMT in the present section. 

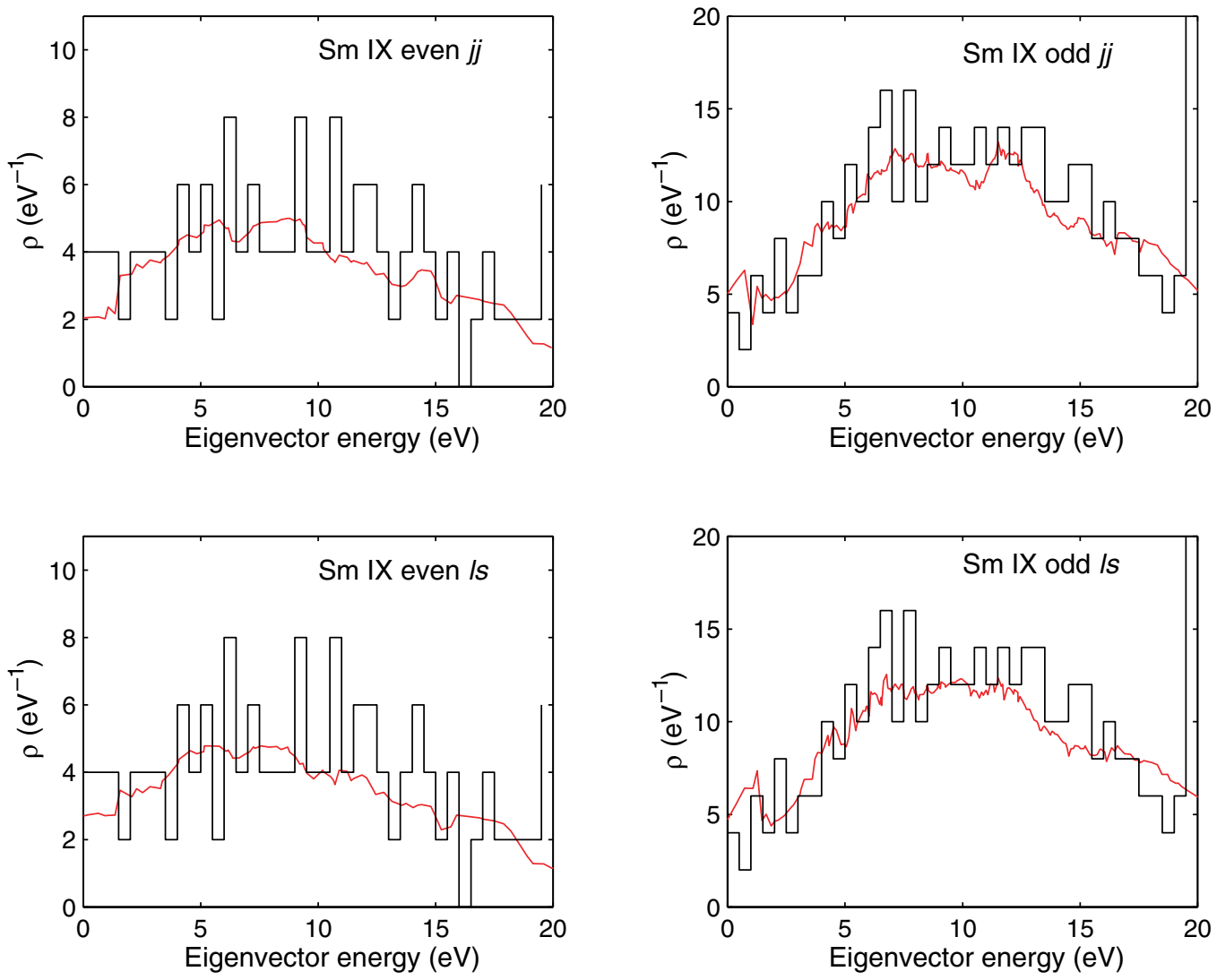

Figure 11. The density of states of Sm IX shown by the histogram and the density of states calculated using $N$ and $\Gamma$ from the Lorentzian curve fit.
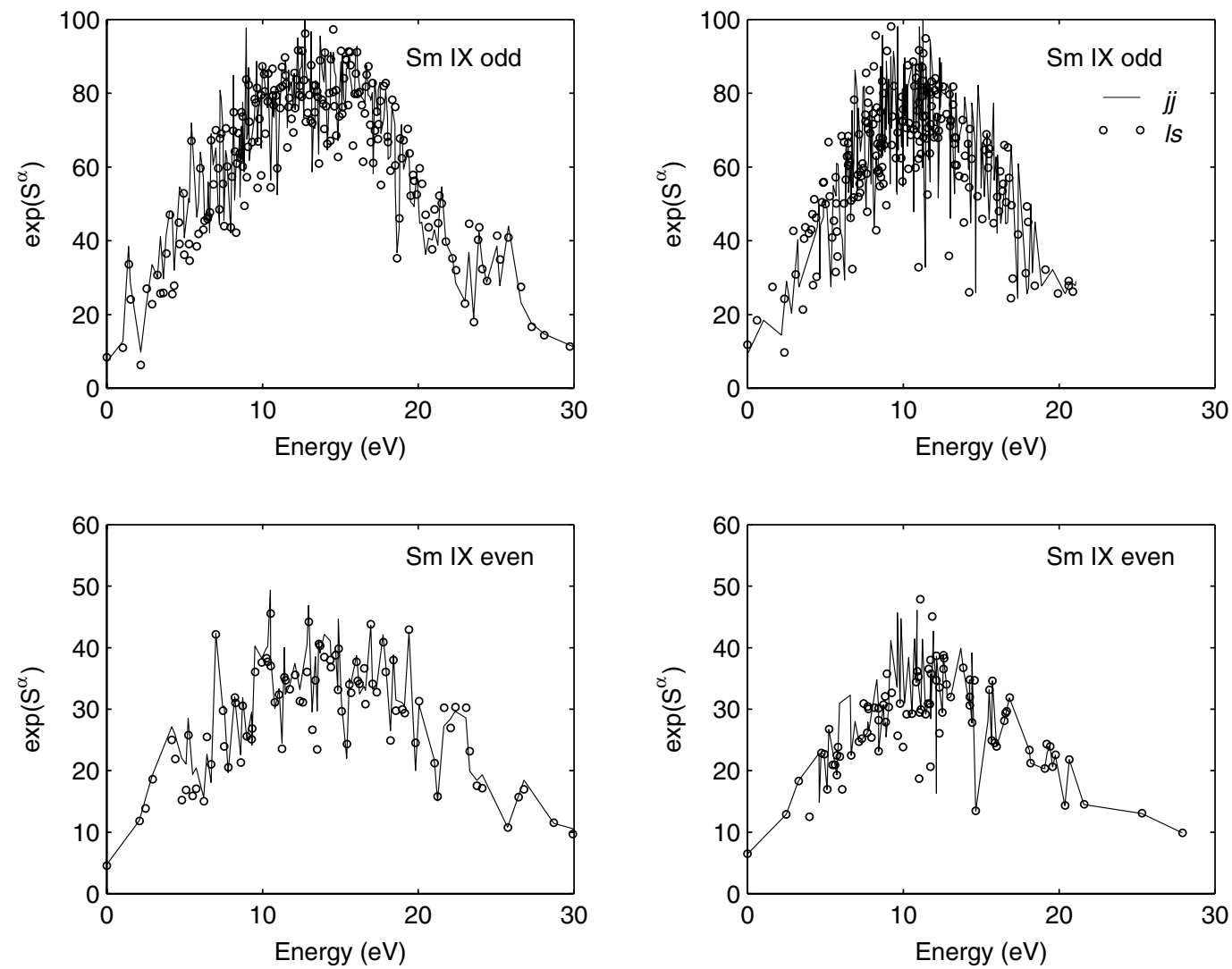

Figure 12. The information length entropy $\exp \left(S^{\alpha}\right)$ for Sm IX in $l s$ and $j j$ coupling schemes. The left-hand columns correspond to the $\exp \left(S^{\alpha}\right)$ of the eigenvectors and the right-hand columns correspond to $\exp \left(S^{\alpha}\right)$ of the basis vectors. 

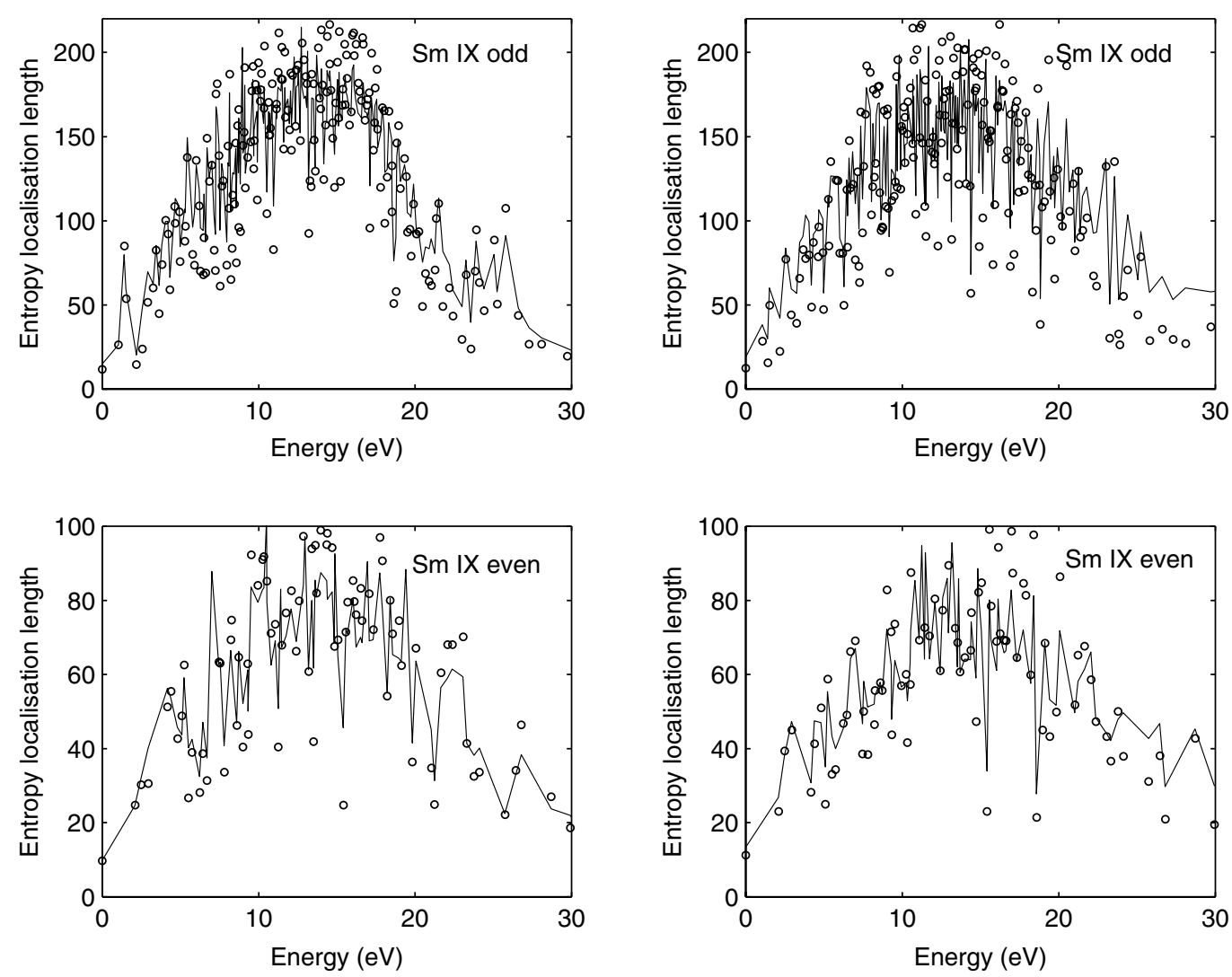

Figure 13. Entropy localization length for Sm IX in $l s$ coupling schemes. The left-hand columns correspond to the eigenvectors whereas the right-hand columns correspond to the basis vectors. The solid line indicates $l_{h}$ whereas the circles correspond to $3.5 \times N P C$.
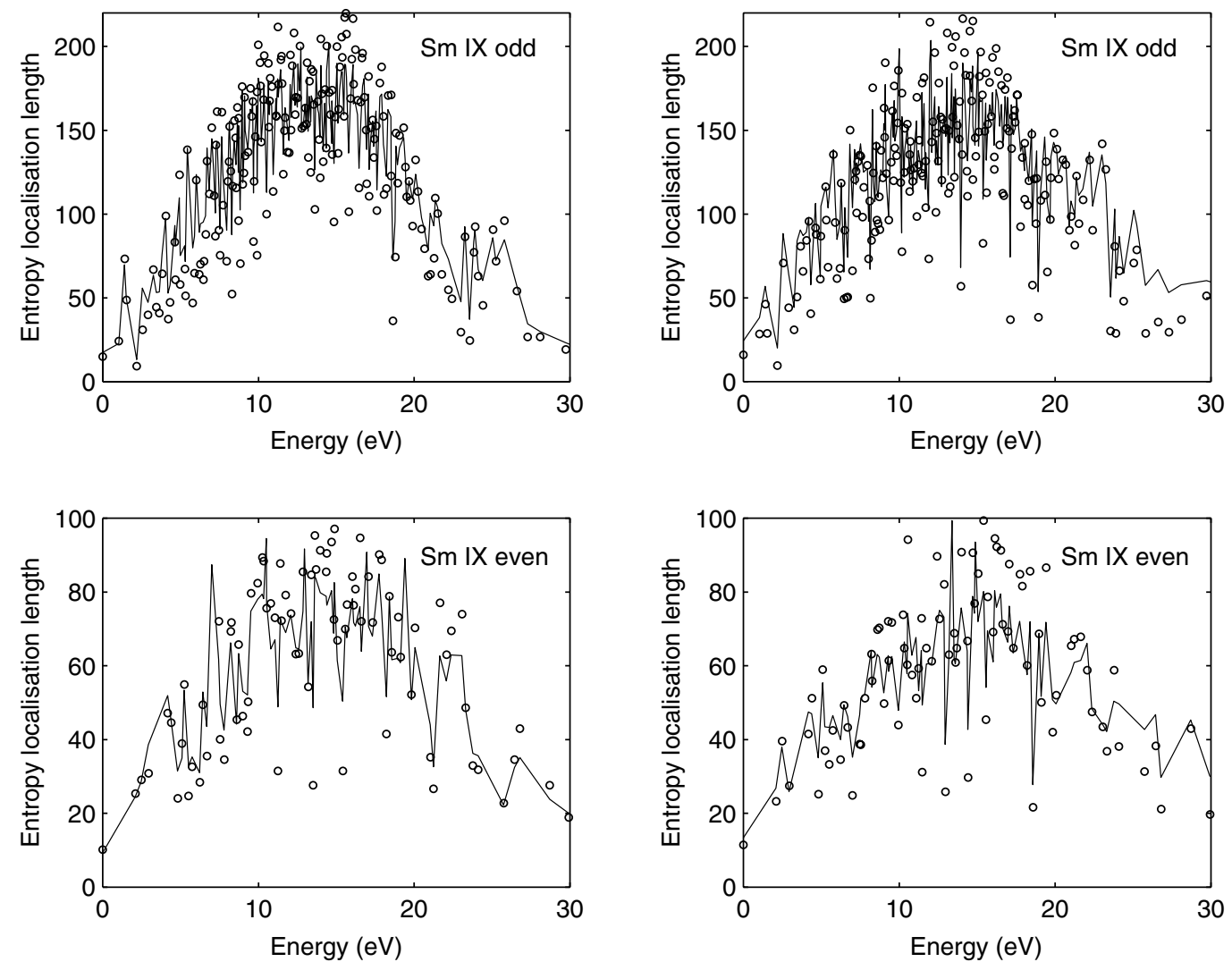

Figure 14. Entropy localization length for Sm IX in $j j$ coupling schemes. The left-hand columns correspond to the eigenvectors whereas the right-hand columns correspond to the basis vectors. The solid line indicates $l_{h}$ whereas the circles correspond to $3.5 \times N P C$. 

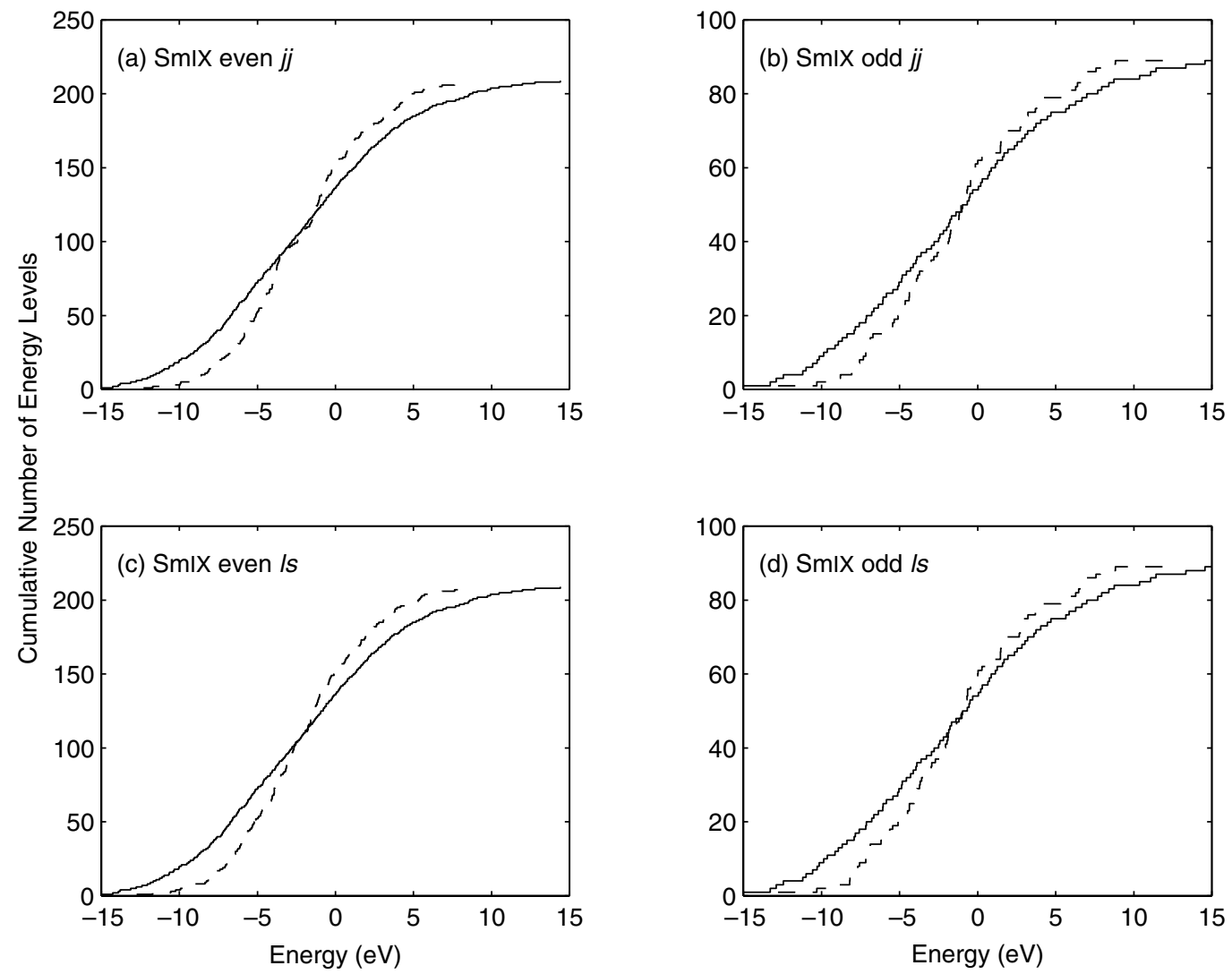

Figure 15. Cumulative number of levels for the CI ( - ) and non-CI eigenvalues (- - - - ) of Sm IX.

Table 7. Mean level spacings of the unperturbed levels $D$ and the perturbed levels $D_{\text {eig }}$ (calculated for the whole of the energy regions).

\begin{tabular}{lll}
\hline & \multicolumn{2}{c}{ Sm IX } \\
\cline { 2 - 3 } & Even & Odd \\
\hline$D(\mathrm{eV})$ & 0.284 & 0.102 \\
$D_{\text {eig }}(\mathrm{eV})$ & 0.348 & 0.143 \\
\hline
\end{tabular}

\subsection{Cumulative number of levels}

In order to examine the effects of CI on the eigenvalues of the system, the cumulative number of levels of Sm IX is shown in figure 15, where the density of states $\rho(E)$ can be estimated as the slope of the curve averaged over the level to level fluctuations of their positions [9]. CI gives rise to level repulsion and a straightening of the cumulative number of energy states as compared to the unperturbed $H_{0}$ energy eigenvalues indicated by the broken curves. CI increases the level spacing of the eigenvalues in both the even and odd case by approximately $6 \%$ and $4 \%$ respectively as can be seen in table 7 .

\subsection{Unfolding the energy levels}

The eigenvalues were unfolded (re-scaled)[37] using the following function for $\bar{N}$, the mean mode number:

$$
\bar{N}\left(\rho_{0}, a, E\right)=\int_{E_{0}}^{E} \rho_{0} \exp \left(a \sqrt{E^{\prime}-E_{0}}\right) \mathrm{d} E^{\prime},
$$

where $\rho_{0}$ and $a$ are treated as curve fitting parameters (values given in table 8 ) for the rising part of the $N(E)$ versus $E$ curve and $E_{0}$ is the energy of the lowest term involved. The curve fitting was only calculated for the energy regions shown in table 8 which were zeroed before the curve fitting procedure. Note that the configurations used in this work are different from those of [25].

Figure 16 shows the mode number $N$ and the mean mode number $\bar{N}$, for even and odd Sm IX, along with the plots of $N$ versus the unfolded energies, which have slopes of $m$ and intercept $c$ given in table $8 . m$ is very close to 1.0 for both even and odd Sm IX which gives the desired average level density (global) of 1.0 for the unfolded energies.

\subsection{Nearest-neighbour spacing distribution}

In [25], the effect of CI and non-CI on the nearest-neighbour spacing (NNS) of Sm IX was highlighted. It was found that CI gave rise to a Wigner NNS distribution, whereas the nonCI gave rise to a Poisson distribution. The NNS distributions were calculated using the unfolded energies and are shown in figure 17. The Wigner (broken line) and Poisson (chain) distributions represent the extremes of classical behaviour, i.e. regular (Poisson) and chaotic (Wigner). The intermediate behaviour observed here and in most real systems can be characterized using the Brody distribution [38, 39]:

$$
P(x, \omega)=\alpha(\omega) \cdot(\omega+1) \cdot x^{\omega} \cdot \mathrm{e}^{-\alpha(\omega) \cdot x^{\omega+1}},
$$

where $\alpha(\omega)=\Gamma\left(\frac{\omega+2}{\omega+1}\right)^{\omega+1}$. A graph of $\ln \left(\ln \left[(1-\Pi(s))^{-1}\right]\right)$ versus $\ln (s)$ is plotted and $\omega$, the Brody parameter, is found 

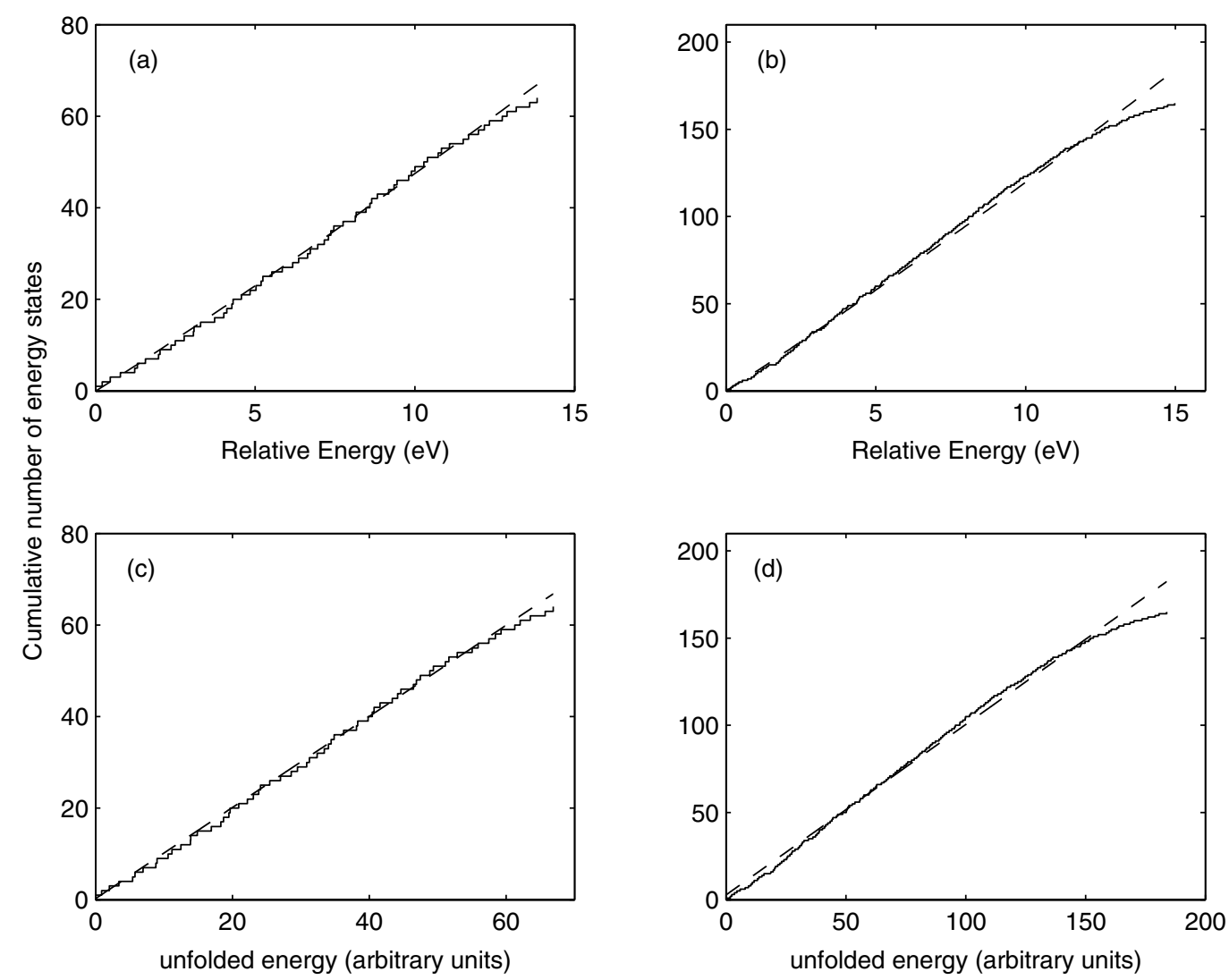

Figure 16. The mode number (full curve) and the mean mode number (broken curve) of even Sm IX ((a) and (c)) and odd Sm IX ((b) and (d)). The mode number and the mean mode number of the unfolded energy eigenvalues are also shown.

Table 8. Various curve fitting parameters of the energy eigenvalue statistics of Sm IX.

\begin{tabular}{lll}
\hline & \multicolumn{2}{c}{ Sm IX } \\
\cline { 2 - 3 } & Even & Odd \\
\hline Unfolded energy & & \\
range $(\mathrm{eV})$ & $-9.139 \rightarrow 4.689$ & $-9.026 \rightarrow 5.954$ \\
$\rho_{0}\left(\mathrm{eV}^{-1}\right)$ & 4.248 & 10.667 \\
$a\left(\mathrm{eV}^{-\frac{1}{2}}\right)$ & 0.052 & 0.054 \\
$m$ & 0.995 & 0.977 \\
$c$ & 0.223 & 2.674 \\
$1+\omega$ & 1.68 & 1.957 \\
$\%$ error on $1+\omega$ & 2.376 & 1.966 \\
$\omega$ & 0.68 & 0.957 \\
Covariance of spacings & -0.1856 & 0.0541 \\
\hline
\end{tabular}

from its slope of $(1+\omega)$ [40] given in table 8 , where $\Pi(s)$ is the cumulative spacing distribution, i.e.

$$
\Pi(s)=\int_{0}^{s} P(x) \mathrm{d} x \equiv 1-\mathrm{e}^{-\alpha(\omega) \cdot s^{\omega+1}} .
$$

Figure 17 shows $\Pi(s)$ and $\ln \left(\ln \left[(1-\Pi(s))^{-1}\right]\right)$ versus $\ln (s)$. Both even and odd configurations give rise to Wigner-like distributions with the odd configuration having a Brody parameter very close to 1 which indicates a GOE Wigner spectrum and chaotic behaviour. A Brody parameter of 0 indicates a Poisson spectrum and regular behaviour. For even Sm IX, $\omega=0.68$ which is indicative of intermediate behaviour. It must be noted that the level statistics are greatly affected by the number of levels and the unfolding procedure. Since the matrix dimension for even Sm IX is very small (91) this may not be a statistically significant set of data. Large fluctuations from the GOE predicted cumulative density of states are observed for even Sm IX which further supports this statement.

\subsection{Covariance of adjacent spacings}

The covariance of adjacent spacings [39, 41, 42]:

$$
\operatorname{cov}\left(s_{n}, s_{n+1}\right)=\frac{\sum_{n=1}^{N}\left(s_{n}-\left\langle s_{n}\right\rangle\right)\left(s_{n+1}-\left\langle s_{n+1}\right\rangle\right)}{\left[\left(\sum_{n=1}^{N}\left(s_{n}-\left\langle s_{n}\right\rangle\right)^{2}\right)\left(\sum_{n=1}^{N}\left(s_{n+1}-\left\langle s_{n+1}\right\rangle\right)^{2}\right)\right]^{\frac{1}{2}}}
$$

tests for local correlations (between levels $E_{i}$ and $E_{i+2}$ ) and is predicted by GOE theory to have a value of -0.27 [43]. The covariance of spacings for even Sm IX is closer to the GOE prediction than the value for odd Sm IX as shown in table 8 . The value for odd Sm IX is very close to the Poisson value of 0 , i.e. no correlations. The value of $\omega=0.957$ and a covariance of approximately 0 for odd Sm IX are suggestive of an uncorrelated Wigner spectrum [44], i.e. level repulsion but with no correlation between $E_{i}$ and $E_{i+2}$. Similar behaviour was reported in [18] for odd Ce. 

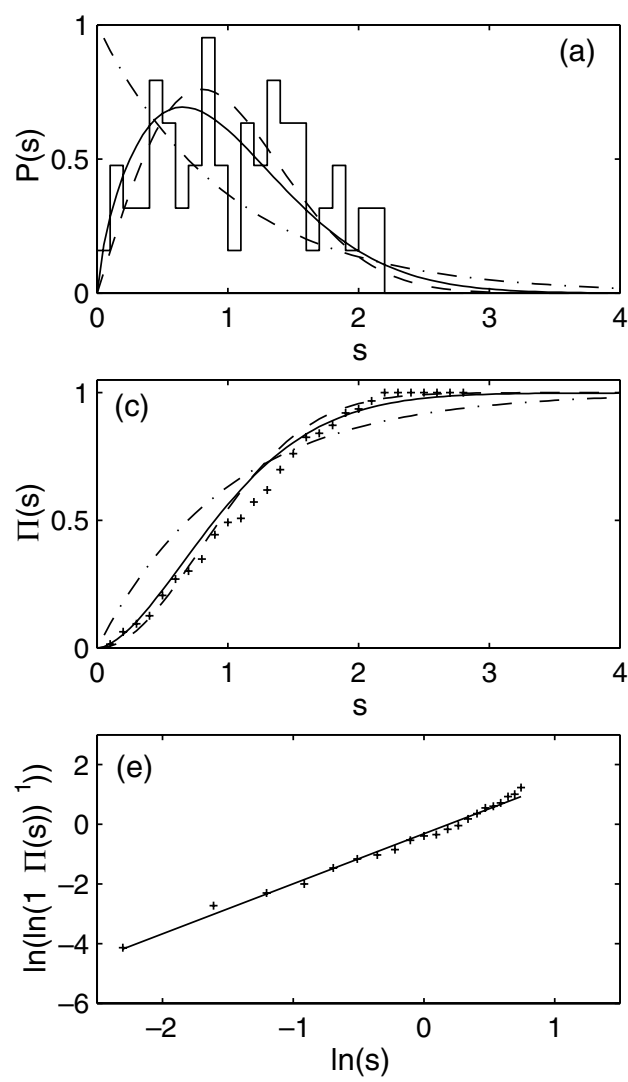
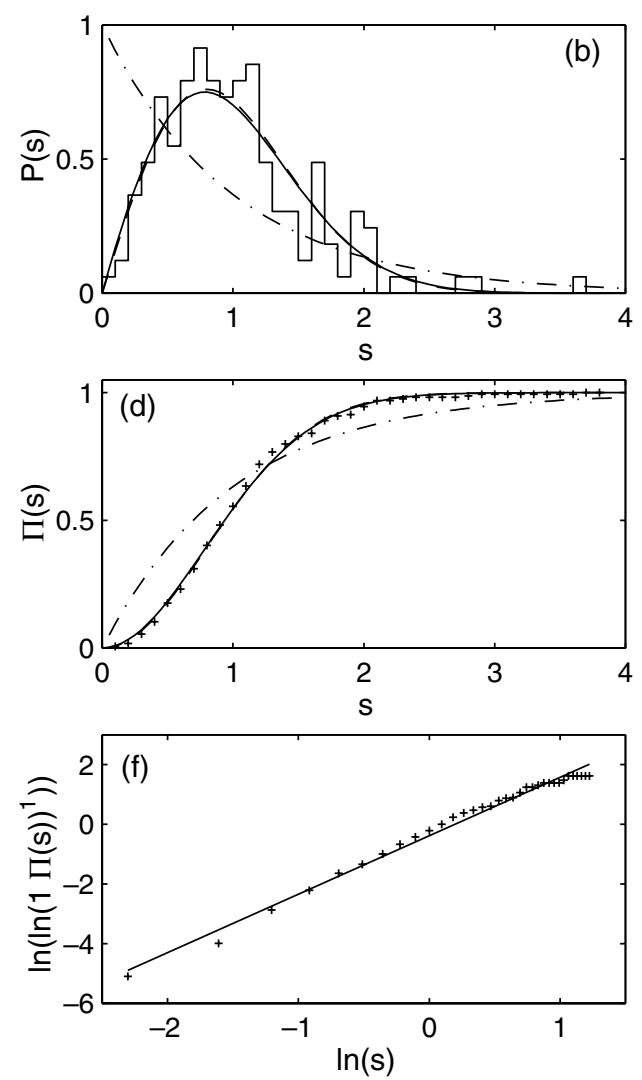

Figure 17. $P(s), \Pi(s)$ and $\ln \left(\ln (1-\Pi(s))^{-1}\right)$ for Sm IX. The left columns correspond to even Sm IX and the right columns correspond to odd Sm IX.

\subsection{Spectral rigidity}

The spectral rigidity $\Delta_{3}(L)$ [45] is defined for the interval $[a, a+L]$ in the sequence $\left\{x_{i}\right\}$ as

$$
\triangle_{3}(a, L)=\frac{1}{L} \min _{A, B} \int_{a}^{a+L}[n(x)-(A x+B)]^{2} \mathrm{~d} x,
$$

where $n(x)$ is the cumulative number of 'states' of the sequence $\left\{x_{i}\right\}$ [46, 47]. For a regular quantum system, the energy levels are distributed randomly and without correlation. A random sequence of uncorrelated levels has a soft character (linear dependence on $L$, i.e. $\Delta_{3}(L)=L / 15$ ). In contrast, the energy levels of a chaotic quantum system exhibit strong correlations owing to the repulsion between the random matrix eigenvalues. A random matrix spectrum has a rigid character (a logarithmic dependence on $L$, i.e. for $L \gg 1, \Delta_{3}(L)=\frac{1}{\pi^{2}}\left(\ln (2 \pi L)+\gamma+1-\frac{5}{4}-\frac{\pi^{2}}{8}\right)$ for GOE matrices, where $\gamma$ is the Euler's constant) [42, 48, 49]. The difference between the two spectra is most pronounced in the $\Delta_{3}(L)$ statistic since this involves correlations between many levels, i.e. they test for long-range spectral fluctuations. The spectral rigidity results are shown in figure 18 , where \langle\rangle indicates spectral averaging.

For both even and odd Sm IX, a rigid spectrum is observed for a limited value of $L$. Even Sm IX shows GOE-like behaviour for $L \leqslant 15$ after which the rigidity gradually decreases. Odd Sm IX shows GOE-like behaviour for $L \leqslant 30$. For odd $\operatorname{Sm~IX,~}\left\langle\Delta_{3}(L)\right\rangle$ appears to saturate for $L \geqslant 30$, an apparent non-universal result due to shorttime periodic orbits in the semiclassical regime [50] and/or the unfolding procedure. For even Sm IX for $L \geqslant 15$ the $\left\langle\Delta_{3}(L)\right\rangle$ approaches the uncorrelated Wigner result $\Delta_{3}(L)=L /[55-(210 / L)]$ for $L>15$ [44]. For odd Sm IX, $\left\langle\Delta_{3}(L)\right\rangle$ approaches the uncorrelated Wigner result for $L>$ 30 until $L \sim 85$ after which the spectral rigidity shows an upbend. Intermediate behaviour between Poisson and GOE is suggestive, classically, of a mixed phase space of regular and chaotic motion [51]. In the limit of large $L \sim 85$, the increase of $\left\langle\Delta_{3}(L)\right\rangle$ exhibited in the odd Sm IX spectrum cannot be explained and seems to be caused by large-scale fluctuations of the cumulative level density.

\subsection{Correlation-hole method}

Leviander et al [52] developed the correlation-hole method for the analysis of long-range correlations. The properly smoothed Fourier transform $C(t)$ of the spectral autocorrelation function maps the long-range correlations onto scales in Fourier space (time domain), i.e. $C(t)$ can be written as the Fourier transform of the autocorrelation function of a spectrum $I(x)$ on the unfolded scale $x$ [53]:

$$
C(t)=\int_{-\infty}^{+\infty} A(r) \mathrm{e}^{2 \pi \mathrm{i} r t} \mathrm{~d} r
$$

where $A(r)=\int_{-\infty}^{+\infty} I\left(R-\frac{r}{2}\right) I\left(R+\frac{r}{2}\right) \mathrm{d} R$ is the autocorrelation function of $I(x)$. In the case of a 'stick' spectrum of equal amplitudes $S(E)=\sum_{\nu} \delta\left(E-E_{\nu}\right)$ then [53]

$$
|C(t)|^{2}=\left|\sum_{j, k} \mathrm{e}^{2 \pi \mathrm{i}\left(E_{j}-E_{k}\right) t}\right|^{2} .
$$



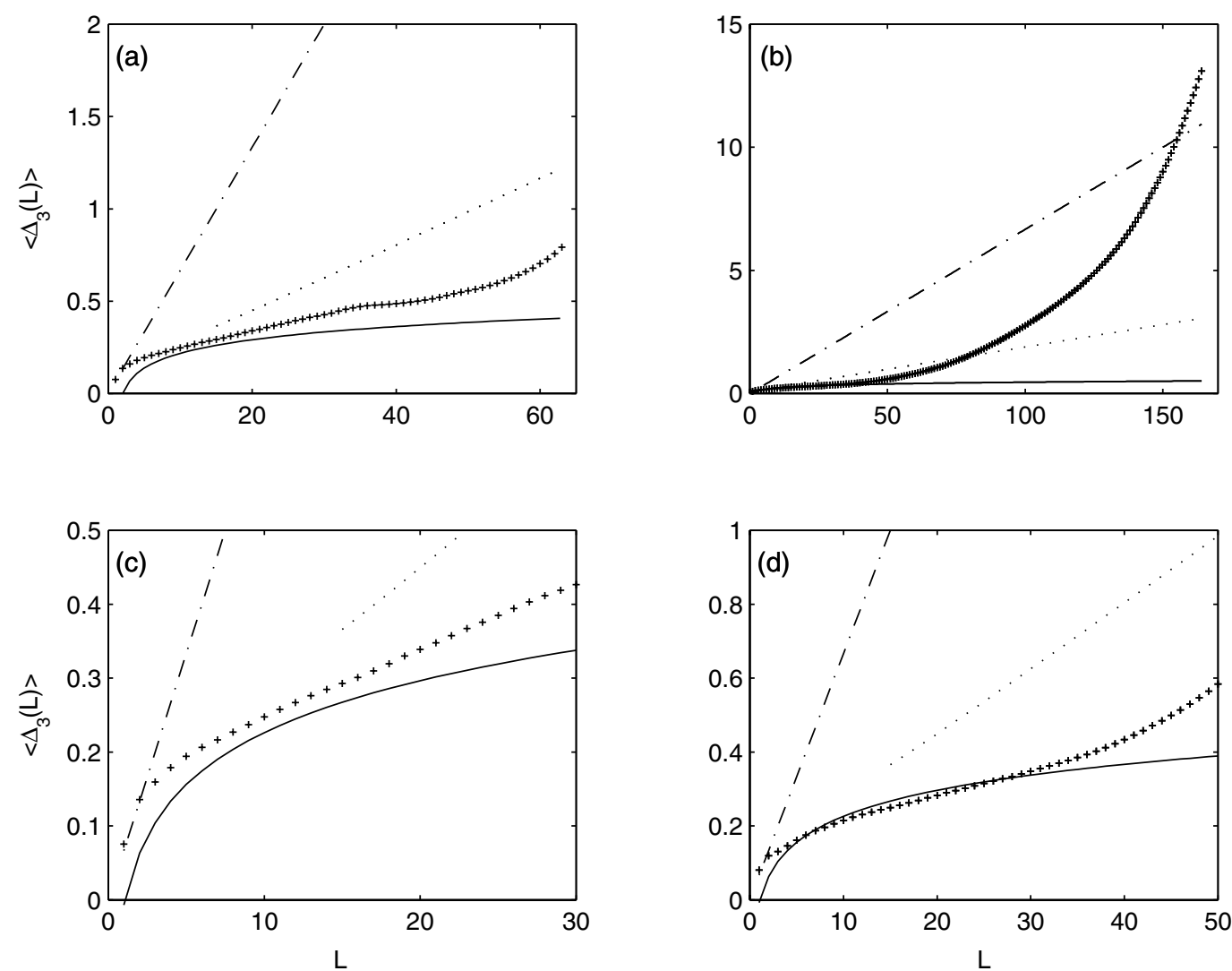

Figure 18. The spectral rigidity of Sm IX. Results are shown for Poisson (chain), GOE (full) and uncorrelated GOE (dotted). The left columns correspond to even Sm IX and the right columns correspond to odd Sm IX.
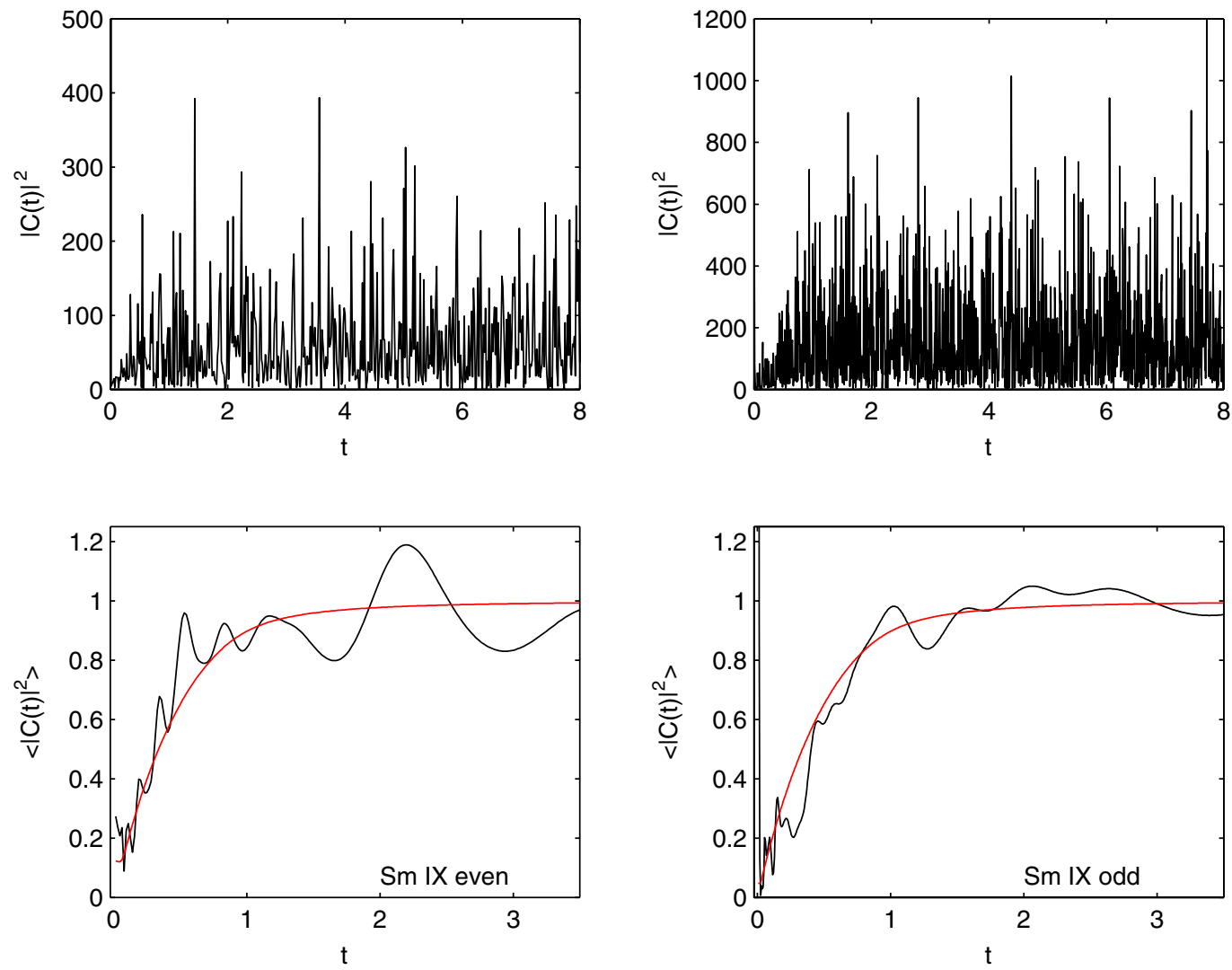

Figure 19. |FourierTransform $\left.\right|^{2}$ and the spectral averaged $\left\langle|C(t)|^{2}\right\rangle$ of the unfolded energies of Sm IX. The left columns correspond to even Sm IX and the right columns correspond to odd Sm IX. 
The ensemble average can be simulated by applying a full Gaussian smoothing procedure to the experimental decay function $|C(t)|^{2}$ [53]. The theoretical $\left\langle|C(t)|^{2}\right\rangle$ is given by [53]

$$
\left\langle|C(t)|^{2}\right\rangle=N^{2}\left(\frac{\sin (\pi N t)}{\pi N t}\right)^{2}+N-N b_{2}(t),
$$

where $b_{2}(t)$ is the two-level form factor $[42,49]$ and $N$ is the number of levels in the spectrum. Chaotic dynamics cause a suppression of the Fourier transform equation (24) near the origin, a so-called 'correlation hole'. For cases intermediate between fully chaotic and regular systems, the correlation hole is less deep and/or abrupt. In particular, a Poisson spectrum should show no correlation hole [52, 54].

The $\mid$ Fouriertransform $\left.\right|^{2},|C(t)|^{2}$ of the unfolded energies is shown in figure 19. The theoretical $\left\langle|C(t)|^{2}\right\rangle$ shown in the figures was also Gaussian smoothed. In both cases, even and odd Sm IX, a correlation hole is present. This hole can indicate the presence of quantum chaos. However, it should be noted that false correlation holes can arise even for regular systems [55], for example, in uncorrelated Wigner spectra. The appearance of the correlation hole as $k$ increases is consistent with the behaviour found for $\left\langle\Delta_{3}(L)\right\rangle$ since small $t$ corresponds to large correlation lengths $L$ in $\left\langle\Delta_{3}(L)\right\rangle$. The presence of highly oscillatory small time behaviour in $\left\langle|C(t)|^{2}\right\rangle$ may indicate the effect of non-universal behaviour in the $\left\langle\Delta_{3}(L)\right\rangle$ statistic. A more plausible explanation for the present case may be due to the finite resolution and/or the unfolding procedure associated with missing energy levels [55].

\subsection{Discussion III}

All of the statistical tests on the energy levels indicate the strong possibility of quantum chaos in odd Sm IX and intermediate behaviour between regular and chaotic regimes for even Sm IX. However, the NNS, $\triangle_{3}(L)$ and $\left\langle|C(t)|^{2}\right\rangle$, are highly dependent on the unfolding procedure which is greatly affected by missing energy levels. A large number of statistical tests is required to search for signatures of quantum chaos, with no individual test being able to provide a decisive test of regular versus chaotic behaviour.

\section{Conclusion}

As has consistently been the case throughout studies on the rare-earth elements, the Hamiltonian matrices once more can best be described by BRM as sparse banded matrices with a leading diagonal. However BRM cannot account for the existence of nonzero off-diagonal elements. We have extended the previous analysis to include an investigation into the behaviour of the eigenstates and found them to be compound atomic states formed by the large mixing of the basis states due to $\mathrm{CI}$ and characterized by the spreading width $\Gamma$ and the localization length. The energy level statistics indicate the strong possibility of quantum chaos, particularly in odd Sm IX and intermediate behaviour between regular and chaotic for even Sm IX, thus reinforcing the conclusion of earlier work and pointing to the universality of behaviour in complex physical systems.

\section{Acknowledgments}

This work was supported by the Irish Science and Technology Agency Enterprise Ireland under research grant no SC/99/206.

\section{References}

[1] Wigner E P 1955 Ann. Math. 62548

[2] Wigner E P 1957 Ann. Math. 65203

[3] Porter C E and Rosenzweig N 1960 Phys. Rev. 1201698

[4] Izrailev F M 1990 Phys. Rep. 196299

[5] Fyodorov Y F and Mirlin A D 1994 Int. J. Mod. Phys. 83795

[6] Casati G, Molinari L and Izrailev F M 1990 Phys. Rev. Lett. 641851

[7] Kús M, Lewenstein M and Haake F 1991 Phys. Rev. A 442800

[8] Fyodorov Y F and Mirlin A D 1991 Phys. Rev. Lett. 672405

[9] Flambaum V V, Gribakina A A, Gribakin G F and Kozlov M G 1994 Phys. Rev. A 50267

[10] Flambaum V V, Gribakina A A and Gribakin G F 1996 Phys. Rev. A 542066

[11] Flambaum V V, Gribakina A A and Gribakin G F 1996 Phys. Rev. E 535729

[12] Flambaum V V, Gribakina A A and Gribakin G F 1998 Phys. Rev. A 58230

[13] Flambaum V V, Gribakina A A, Gribakin G F and Harabati C 2002 Phys. Rev. A 66012713

[14] Gribakina A A, Flambaum V V and Gribakin G F 1995 Phys. Rev. E 525667

[15] Gribakina A A and Gribakin G F 1996 J. Phys. B: At. Mol. Opt. Phys. 29 L809

[16] Zelevinsky V G, Brown B A, Fraizer N and Horoi M 1996 Phys. Rep. 27685

[17] Cummings A, O'Sullivan G and Heffernan D M 2001 J. Phys. B: At. Mol. Opt. Phys. 343407

[18] Cummings A, O'Sullivan G and Heffernan D M 2001 J. Phys. B: At. Mol. Opt. Phys. 343447

[19] Cummings A, O'Sullivan G, Hanan W G and Heffernan D M 2001 J. Phys. B: At. Mol. Opt. Phys. 342547

[20] Carroll P K and O'Sullivan G 1982 Phys. Rev. A 25275

[21] O’Sullivan G 1982 J. Phys. B: At. Mol. Phys. 163291

[22] Curtis L J and Ellis D G 1980 Phys. Rev. Lett. 452099

[23] Curtis L J 1986 J. Opt. Soc. Am. B 31102

[24] Kaufman V et al 1990 Phys. Scr. 42705

[25] O'Sullivan G et al 1999 J. Phys. B: At. Mol. Opt. Phys. 321893

[26] Carroll P K, Kennedy E T and O’Sullivan G 1980 Appl. Opt. 191454

[27] Kilbane D, Cummings A, McGuinness C, Murphy N and O'Sulivan G 2002 J. Phys. B: At. Mol. Opt. Phys. 35309

[28] Cowan R D 1981 The Theory of Atomic Structure and Spectra (Berkeley, CA: University of California Press)

[29] Sugar J and Kaufman V 1980 Phys. Rev. A 212096

[30] Izrailev F M 2000 Proc. Int. School of Physics 'Enrico Fermi', Course CXLIII, Varenna 20-30 July ed G Casati, I Guarneri and U Smilansky (Amsterdam: IOS Press) pp 371-430

[31] Fyodorov Y F, Chubykalo O, Izrailev F M and Casati G 1996 Phys. Rev. Lett. 761603

[32] Wang W, Izrailev F M and Casati G 1998 Phys. Rev. E 57323

[33] Bohr A and Mottelson B R 1969 Nuclear Structure vol I (Reading, MA: Benjamin)

[34] Yonezawa F 1980 J. Non-Cryst. Solids 35-36 29

[35] Reichl J 1988 Europhys. Lett. 6669

[36] Zelevinsky V G 1993 Nucl. Phys. A 555109

[37] Friedrich H 1991 Theoretical Atomic Physics (Berlin: Springer)

[38] Brody T A 1973 Lett. Nuovo Cimento 71482

[39] Connerade J P, Grant I P, Marketos P and Oberdisse J 1995 J. Phys. B: At. Mol. Opt. Phys. 282539 
[40] Badrinarayanan R and José J V 1995 Quantum Chaos between Order and Disorder ed G Casati and B V Chirikov (Cambridge: Cambridge University Press)

[41] Porter C E 1965 Statistical Theories of Spectra: Fluctuations (New York: Academic)

[42] Mehta M L 1991 Random Matrices 2nd edn (New York: Academic)

[43] Camarda H S and Georgopulos P D 1983 Phys. Rev. Lett. 50492

[44] Camarda H S et al 1972 Statistical Properties of Nuclei ed J B Garg (New York: Plenum)

[45] Dyson F J and Metha M L 1963 J. Math. Phys. 4701

[46] Bohigas O and Giannoni M J 1975 Ann. Phys. (N. Y.) 89393
[47] Ormond W E and Broglia R A 1992 Phys. Rev. C 465

[48] Pandey A 1979 Ann. Phys. 119170

[49] Guhr T, Müller-Groeling A and Weidenmüller H A 1998 Phys. Rev. 299190

[50] Berry M 1985 Proc. R. Soc. A 400229

[51] Percival I C 1977 Adv. Chem. Phys. 361

[52] Leviander L, Lombardi M, Jost R and Pique J P 1986 Phys. Rev. Lett. 562449

[53] Belon A, Jost R and Lombardi M 1991 J. Chem. Phys. 955701

[54] Lombardi M, Bohigas O and Seligman T H 1994 Phys. Lett. B 324263

[55] Lombardi M, Labastie P and Bordas M C 1979 J. Chem. Phys. 893479 